\title{
High-pressure phase transformations, pressure-induced amorphization, and polyamorphic transition of the clathrate $\mathbf{R b}_{6.15} \mathrm{Si}_{46}$
}

\author{
D. Machon, ${ }^{1}$ P. Toulemonde, ${ }^{1,2}$ P. F. McMillan, ${ }^{3}$ M. Amboage,,${ }^{4,5}$ A. Muñoz, ${ }^{6}$ P. Rodríguez-Hernández, ${ }^{6}$ and A. San Miguel ${ }^{1}$ \\ ${ }^{1}$ LPMCN, Université Lyon-I, Bâtiment Léon Brillouin, 43 Boulevard du 11 Novembre 1918, F-69622 Villeurbanne Cedex, France \\ ${ }^{2}$ Institut Néel, CNRS and Université Joseph Fourier, 25 Avenue des Martyrs, BP 166, F-38042 Grenoble Cedex 9, France \\ ${ }^{3}$ Department of Chemistry and Materials Chemistry Centre, Christopher Ingold Laboratories, University College London, \\ 20 Gordon Street, London WC1H OAJ, United Kingdom \\ ${ }^{4}$ European Synchrotron Radiation Facility, BP 220, F-38043 Grenoble Cedex 9, France \\ ${ }^{5}$ Diamond Light Source, Harwell Science and Innovation Campus, Didcot, Oxfordshire OX11 ODE, United Kingdom* \\ ${ }^{6}$ Departamento de Física Fundamental II, MALTA Consolider Team, Universidad de La Laguna, La Laguna, 38205 Tenerife, Spain
}

(Received 17 November 2008; revised manuscript received 3 March 2009; published 4 May 2009)

\begin{abstract}
The type-I clathrate $\mathrm{Rb}_{6.15} \mathrm{Si}_{46}$ with partly empty cage sites has been studied up to $36 \mathrm{GPa}$ using Raman spectroscopy, synchrotron x-ray diffraction in diamond-anvil cells, and ab initio total-energy and latticedynamics calculations. A first phase transition is observed at $13 \pm 1 \mathrm{GPa}$ and a "volume collapse" transition within the clathrate structure is then observed at $24 \pm 1 \mathrm{GPa}$. Pressure-induced amorphization into a highdensity amorphous (HDA) state occurs above $P=33 \pm 1$ GPa. The HDA form transforms into a low-density amorphous polymorph during decompression. During the compression study using angle dispersive synchrotron $\mathrm{x}$-ray diffraction techniques we measured bulk modulus parameters for rocksalt-structured TaO, included adventitiously in the clathrate sample $\left[K_{0}=293(3) \mathrm{GPa}\right.$ and $\left.K_{0}^{\prime}=5.4(3)\right]$.
\end{abstract}

DOI: 10.1103/PhysRevB.79.184101

PACS number(s): 61.50.Ks, 64.60.My

\section{INTRODUCTION}

Since the first syntheses of alkali-doped Si and Ge "semiconductor clathrate" phases by the metastable thermal decomposition of Zintl compounds such as $\mathrm{NaSi}$ or $\mathrm{NaGe}$ (Refs. 1-3) or by direct reaction between the elements, ${ }^{4}$ there has been increasing interest in this unusual family of solidstate materials. ${ }^{5,6}$ Analogous clathrate structures are now known to occur with group 13-15 elements, halogens, chalcogenides, transition-metal atoms substituting for $\mathrm{Si}$ or $\mathrm{Ge}$ on framework sites, and various alkali or alkaline-earth atoms, halogens, chalcogenides, transition metals, and lanthanides occupying "guest" sites. ${ }^{5-7}$ The semiconductor clathrates correspond to open framework structures formed by assemblage of $T_{20}, T_{24}$, or $T_{28}$ polyhedral cages, where $T=\mathrm{Si}$, Ge etc. atoms are in slightly distorted tetrahedral coordination with $s p^{3}$ bonding. The cages are fully or partly filled with guest atoms. Clathrate materials have received considerable attention because of their unusual and potentially useful electronic, ${ }^{8}$ optical, ${ }^{9}$ mechanical, ${ }^{10}$ and thermoelectric ${ }^{11}$ properties or as electron emitters ${ }^{12}$ between others. Depending upon the degree of guest atom filling vs framework site occupancy, they exhibit electrical behavior ranging from semiconducting to metallic. Between others, clathrates in the systems $\left(\mathrm{Na}_{1-x} \mathrm{Ba}_{x}\right)_{8} \mathrm{Si}_{46}$ and $\left(\mathrm{Ba}_{1-x} \mathrm{Sr}_{x}\right)_{8} \mathrm{Si}_{46}$ are superconducting with $T_{c}$ between 4 and $8 \mathrm{~K}^{13-15}$ The relationship between the electronic and vibrational properties of the framework vs guest atom sublattices is of particular interest giving rise to unique thermoelectric behavior.

We will adopt here the basic clathrate structure description based on three clathrate types which map on to structures formed by the $\mathrm{H}_{2} \mathrm{O}$-based clathrate hydrates. ${ }^{16}$ More complete classifications have been also proposed to describe symmetry variations in ternary group-14 clathrates. ${ }^{17}$ The type-I clathrate (space group $\operatorname{Pm} \overline{3} n$ ) contains two $\mathrm{Si}_{20}$ and six $\mathrm{Si}_{24}$ polyhedral cages and it provides a total of eight (two small and six large) occupancy sites for guest atoms. That gives rise to compositions such as $\mathrm{Na}_{8} \mathrm{Si}_{46}$ if all guest sites are filled or $\mathrm{Rb}_{6} \mathrm{Si}_{46}$ if only the larger sites are occupied. These compounds are metallic because of the contribution from the alkali atom electrons to the conduction band. ${ }^{18}$

There have been many studies of high-pressure transformations among the stable and metastable polymorphs of elemental Si. ${ }^{19}$ The diamond-structured polymorph of silicon transforms into the metallic $\beta$-Sn structured polymorph with the atoms in distorted octahedral coordination at $10.5 \mathrm{GPa},{ }^{20}$ followed by further transitions into dense metallic phases with the atoms in higher coordination at higher pressures. ${ }^{21}$ A guest-free variety of the type-II clathrate $\mathrm{Si}_{136}$ has been prepared that constitutes a low-density allotrope of the element and that is metastable relative to the diamond structure at ambient pressure. ${ }^{5,9,22}$ Based on the projected intersection of free-energy $(G)$ curves, it was initially predicted that $\mathrm{Si}_{136}$ would transform into the $\beta$-Sn structure at a lowered pressure $(P \sim 3-4 \mathrm{GPa})$ compared to the diamond phase. ${ }^{9}$ However, that result was not observed experimentally probably due to kinetic effects associated with $\mathrm{Si}-\mathrm{Si}$ bond breaking and a direct transition to the $\beta$-Sn polymorph was observed at $P \sim 8-10$ GPa using energy-dispersive synchrotron $\mathrm{x}$-ray diffraction in a diamond-anvil cell. ${ }^{23,24}$ A recent study using Raman spectroscopy has found that the situation is more complex and that at least one intermediate metastable phase is formed during the compression and collapse of the clathrate structure..$^{25}$

Several studies have addressed the high-pressure behavior of clathrates with filled guest cages. $\mathrm{Na}_{8} \mathrm{Si}_{46}$ apparently undergoes pressure-induced decomposition above $P=14 \mathrm{GPa}$, where characteristic peaks of the metallic hexagonal structure of Si begin to appear in the x-ray diffraction pattern. ${ }^{5,26}$ However, type-I clathrates with larger guest atoms 
$\left[\mathrm{K}_{8} \mathrm{Si}_{46},{ }^{27,28} \mathrm{Ba}_{8} \mathrm{Si}_{46},{ }^{29,30}\right.$ and $\mathrm{I}_{8} \mathrm{Si}_{44} \mathrm{I}_{2}$ (Refs. 5 and 31 ) $]$ exhibit an unusual "volume collapse" transition in this pressure range while apparently retaining the same cubic clathrate crystal structure, followed by pressure-induced amorphization at higher pressure. That behavior and its dependence on the cage site occupancy are not well understood.

The clathrate compound $\mathrm{Rb}_{6.15} \mathrm{Si}_{46}$ is a particularly interesting case for study because the six $\mathrm{Si}_{24}$ cages are filled with the large $\mathrm{Rb}$ guest atoms (occupancy=0.952), but the occupancy of the smaller $\mathrm{Si}_{20}$ cages is substantially smaller than unity (occupancy $=0.218$ ). ${ }^{9}$ It therefore constitutes a useful case to examine the different roles played by filling the two types of nanocages in determining the stability, mechanical properties, and pressure behavior of type-I clathrates. Here we use a combination of experimental (synchrotron angledispersive x-ray diffraction and Raman scattering) and $a b$ initio calculation approaches to study the high-pressure behavior of the type-I clathrate $\mathrm{Rb}_{6.15} \mathrm{Si}_{46}$.

\section{EXPERIMENTAL AND COMPUTATIONAL METHODS}

The synthesis and characterization of $\mathrm{Rb}_{6.15} \mathrm{Si}_{46}$ were described previously and here we used samples obtained from that study. ${ }^{9}$ The $\mathrm{Rb}_{6.15} \mathrm{Si}_{46}$ was synthesized from mixtures of elemental $\mathrm{Rb}$ and $\mathrm{Si}$ at $666^{\circ} \mathrm{C}$ in a Ta capsule and sealed inside a steel bomb for $24 \mathrm{~h}$. Excess alkali was removed from the silicide by evaporation at $250{ }^{\circ} \mathrm{C}$ under a vacuum of $\sim 10^{-5}$ Torr. The silicon clathrate sample was then obtained by evaporating the alkali atoms between 425 and $475^{\circ} \mathrm{C}$ at a pressure of $\sim 10^{-6}$ Torr. Prior to carrying out high-pressure experiments, the sample was stored in a glove box $\left(<10 \mathrm{ppm} \mathrm{O}_{2} / \mathrm{H}_{2} \mathrm{O}\right)$ at room temperature. Aliquots were removed from the sample for loading into diamondanvil cells or to be carried to the synchrotron.

High-pressure in situ synchrotron $\mathrm{x}$-ray diffraction experiments were carried out using a He gas membrane-driven diamond-anvil cell with diamonds having a culet size of $350 \mu \mathrm{m}$. Samples were loaded using nitrogen as a pressuretransmitting medium and the pressures were determined using the ruby fluorescence method. ${ }^{32}$ Pressure was increased by steps of 1-2 GPa and the system was left to equilibrate for 15-30 $\mathrm{min}$ at each pressure.

Synchrotron radiation measurements were performed at the European Synchrotron Radiation Facility (ID9A) via angle-dispersive diffraction techniques using monochromatic radiation $(\lambda=0.412475 \AA)$. Diffraction patterns were collected using image plate detection. The sample-to-detector distance and the image plate orientation angles were calibrated using a crystalline Si standard. The two-dimensional diffraction images were analyzed using the FIT2D software, yielding one-dimensional intensity vs diffraction angle $2 \theta$ patterns. $^{33}$

High-pressure Raman-scattering experiments were carried out using a cylindrical diamond-anvil cell with lowfluorescence diamonds having a culet size of $200 \mu \mathrm{m}$. Pure polycrystalline $\mathrm{Rb}_{6.15} \mathrm{Si}_{46}$ was loaded into a $90 \mu \mathrm{m}$ hole drilled in a Re gasket. The pressure-transmitting medium argon was cryogenically loaded as a fluid into the cell and used as a nearly hydrostatic medium during pressurization experi- ments. Several ruby chips were distributed throughout the sample chamber and the pressures were determined using the ruby fluorescence method. ${ }^{32}$ A nonhydrostatic pressurization experiment was also carried out without any pressuretransmitting medium using the same experimental configuration.

Raman spectra were obtained using a home-built highthroughput optical system based on Kaiser optical notch filters and an Acton 300i spectrograph with sensitive charge coupled device (CCD) detection. ${ }^{34}$ Spectra were excited using $514.5 \mathrm{~nm}$ radiation from an air-cooled $\mathrm{Ar}^{+}$laser. The beam was focused on to the sample using a Mitutoyo $50 \times$ objective with beam diameter $\sim 2 \mu \mathrm{m}$ at the sample. The backscattered light was collected using the same lens.

Total energy and lattice dynamics calculations were carried out within the framework of density-functional theory (DFT) (Ref. 35) and the pseudopotential method using the Vienna $a b$ initio simulation package (VASP) of which a detailed description of the methodology can be found in Ref. 36 and references therein. The exchange and correlation energies were initially formulated within the generalized gradient approximation (GGA) according to the Perdew-Burke-Ernzerhof ${ }^{37}$ (PBE) prescription. The projector-augmented wave (PAW) scheme $^{38}$ was adopted and the semicore $4 s$ electrons of $\mathrm{Rb}$ were dealt with explicitly in the calculations. The set of plane waves used extended up to a kinetic-energy cutoff of $350 \mathrm{eV}$. This cutoff was required to treat the atoms within the PAW scheme to ensure highly converged results. The Monkhorst-Pack ${ }^{39}$ grid used for Brillouin-zone integrations ensured highly converged results (to about $1 \mathrm{meV}$ per formula unit). We tested different meshes for the $\mathrm{Rb}_{x} \mathrm{Si}_{46}$ analyzed structures. At each selected volume, the structures were fully relaxed to their equilibrium configuration through the calculation of the forces on atoms and the stress tensor (see Ref. 40). In the relaxed equilibrium configuration, the forces are less than $0.002 \mathrm{eV} / \AA$ and the deviation of the stress tensor from a diagonal hydrostatic form is less than $1 \mathrm{kbar}(0.1 \mathrm{GPa})$. The highly converged results for the forces are required for the calculation of the dynamical matrix using the direct force-constant approach (or supercell method). ${ }^{41}$ The construction of the dynamical matrix at the $\Gamma$ point is particularly simple and involves separate calculations of the forces in which a fixed displacement from the equilibrium configuration of the atoms within the primitive unit cell is considered. Symmetry aids in the computations by reducing the number of such independent distortions and the amount of computational effort required. Diagonalization of the dynamical matrix provides both the frequencies of the normal modes and their polarization vectors. It allows us to identify the irreducible representation and the character of the phonon modes at the zone center.

\section{RESULTS}

\section{A. X-ray diffraction}

The x-ray diffraction pattern of the $\mathrm{Rb}_{6.15} \mathrm{Si}_{46}$ clathrate compound at ambient pressure is shown in Fig. 1. The main peaks can be assigned and indexed unambiguously as the clathrate type-I structure (space group: $P m \overline{3} n$, No. 223). Us- 


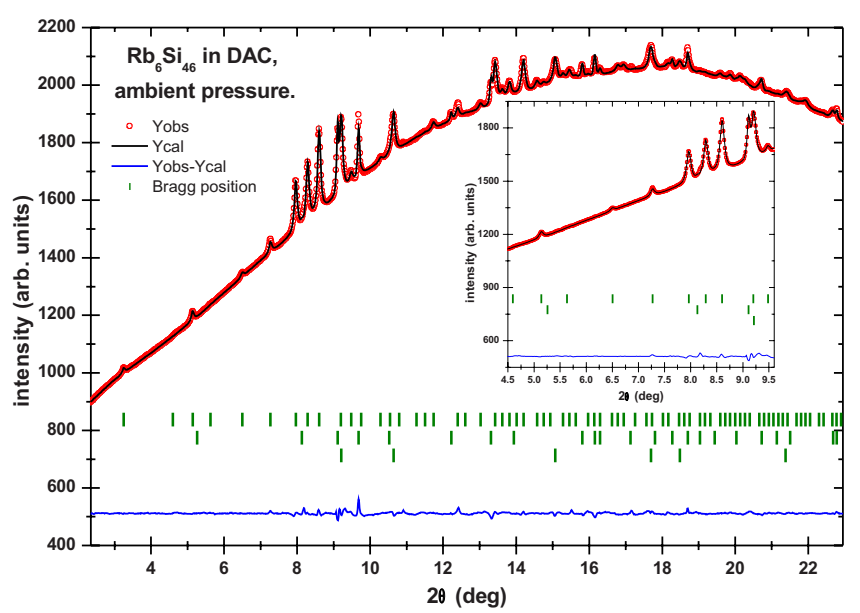

FIG. 1. (Color online) X-ray diffraction pattern including Rietveld refinement of the sample at room pressure in the diamondanvil cell. Three phases are present: the type-I clathrate $\mathrm{Rb}_{6.15} \mathrm{Si}_{46}$ $(P m \overline{3} n)$, the $\varepsilon$ phase of $\mathrm{TaN}(P 6 \overline{2} m)$, and $\mathrm{TaO}$ with rocksalt structure $(F m \overline{3} m)$.

ing Rietveld refinement, the cell parameter was 10.286(1) $\AA$ and the occupancy of the $\mathrm{Si}_{20}$ cages by $\mathrm{Rb}$ atoms was found to be $24 \%$, in agreement with the previous determination. ${ }^{9}$ However, we also observed a series of additional peaks in our diffraction pattern that were due to small amounts of (a) $\varepsilon$-TaN [space group: $P 6-2 m$, No. 189 with cell parameters $a=5.1938(2) \AA$ and $c=2.9101(2) \AA$ (Ref. 42) $]$ and (b) $\mathrm{TaO}$ with the rocksalt structure [space group: $F m-3 m$, No. 225 with cell parameter $a=4.4484(2) \AA]$. These impurity phases were introduced adventitiously during grinding the samples for synchrotron diffraction experiments in the diamond-anvil cell: the agate mortar had previously been used to grind samples of the ultrahard material $\varepsilon$-TaN: some grains remained embedded in the mortar and partly oxidized in air to give $\mathrm{TaO}$; both phases were then transferred into our clathrate sample. From Rietveld analysis we estimate the $\varepsilon$-TaN and $\mathrm{TaO}$ concentrations in mass as $5.8(2) \%$ and $8.4(2) \%$, respectively. The presence of these phases in our sample did not affect the interpretation of the synchrotron $\mathrm{x}$-ray results on the $\mathrm{Rb}_{6.15} \mathrm{Si}_{46}$ clathrate sample: in fact, they provided a useful internal standard for the analysis of linewidths and relative intensities of clathrate features in our high-pressure $\mathrm{x}$-ray diffraction measurements. In addition, our data allowed us to obtain a value for the compressibility of $\mathrm{TaO}$.

In Fig. 2 we show selected diffraction patterns as a function of pressure. The $\mathrm{TaN}$ and $\mathrm{TaO}$ peaks are marked. The unit-cell parameters for all three phases were obtained at each pressure by LeBail refinements using the FULLPROF software, ${ }^{43}$ allowing us to determine relative volume $V / V_{0}$ values. A plot of $V / V_{0}$ as a function of pressure for $\mathrm{Rb}_{6.15} \mathrm{Si}_{46}$ is shown in Fig. 3 using 32 peaks for $\mathrm{Rb}_{6.15} \mathrm{Si}_{46}$ to carry out the global LeBail refinement. A change in slope is clearly observed at $24 \pm 1 \mathrm{GPa}$ marking the presence of a "volume collapse" transition as previously observed for $\mathrm{K}-, \mathrm{Ba}-$, and I-containing clathrates. ${ }^{5,27-31}$ As noted for the other clathrates, there are no obvious changes in the number or relative intensities of peaks in the x-ray diffraction pattern and none of the peaks exhibit any splitting, so it is proposed that the

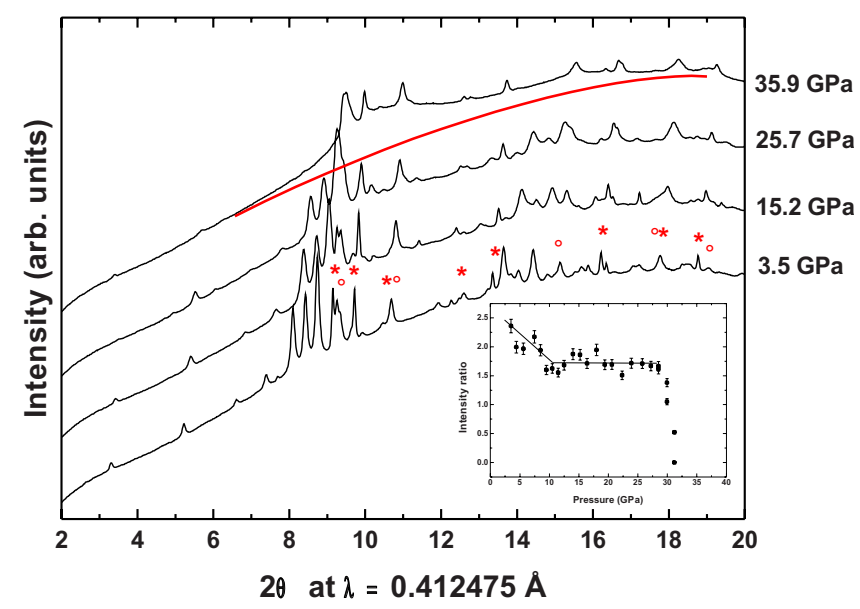

FIG. 2. (Color online) Selected x-ray diffraction patterns with increasing pressure. The peaks due to the $\varepsilon$-TaN phase and $\mathrm{TaO}$ are identified with asterisks and open circles, respectively. At pressure higher than $33 \mathrm{GPa}$ the diffraction peaks from the clathrate $\mathrm{Rb}_{6.15} \mathrm{Si}_{46}$ disappear and are replaced by a broad amorphous signal indicating the occurrence of pressure-induced amorphization. The additional peaks are due to crystalline $\mathrm{TaN}$ and $\mathrm{TaO}$. An estimated background line is drawn to show the likely amorphous contribution to the $\mathrm{x}$-ray scattering at high pressure. Inset: intensity ratio between the (320) clathrate peak and the (200) TaO peak used as a standard. A small decrease of this ratio is observed between ambient pressure and $10 \mathrm{GPa}$ followed by a sudden decrease of the $\mathrm{Rb}_{6.15} \mathrm{Si}_{46}$ peak intensity with a complete disappearance by $33 \mathrm{GPa}$ that indicates amorphization of the clathrate structure.

transition is "homothetic" or isostructural. At $29 \pm 1 \mathrm{GPa}$, an abrupt decrease in intensity of the $\mathrm{Rb}_{6.15} \mathrm{Si}_{46}$ Bragg peaks is observed, relative to the $\mathrm{TaO}$ features that provide a useful internal intensity standard (Fig. 2 inset). By $33 \pm 1 \mathrm{GPa}$, the clathrate peaks have completely disappeared and are replaced by a broad amorphous scattering feature, indicating that pressure-induced amorphization has occurred (Fig. 2). There is no evidence for the appearance of any high-pressure metallic $\mathrm{Si}$ or $\mathrm{Rb}$ phases so we conclude that the amorphous material has $\mathrm{Rb}_{6.15} \mathrm{Si}_{46}$ composition.

$\mathrm{X}$-ray diffraction patterns obtained during decompression are shown in Fig. 4. There is no evidence for recrystallization and an amorphous form of $\mathrm{Rb}_{6.15} \mathrm{Si}_{46}$ is recovered to approximately ambient pressure. During decompression, the main amorphous diffraction feature shifts approximately linearly to lower $2 \theta$ values (i.e., larger $d$ spacing). We could not carry out any more detailed analysis of the amorphous scattering as done in Ref. 44 because of the scattering contribution of the $\mathrm{N}_{2}$ pressurization medium, the presence of strong Bragg diffraction from the $\mathrm{TaN} / \mathrm{TaO}$ impurity/standards, and the lack of proper background pattern recorded during the experiment.

The $V / V_{0}$ data for $\mathrm{Rb}_{6.15} \mathrm{Si}_{46}$ upon compression in the low-pressure range $0.1-24 \mathrm{GPa}$ were initially fitted using a third-order Birch-Murnaghan equation of state model to obtain the bulk modulus $K_{0}$ and its pressure derivative $K_{0}^{\prime}$ for the clathrate phase. The values obtained by this procedure were $K_{0}=59(1) \mathrm{GPa}$ and $K_{0}^{\prime}=7.2(2)$, respectively. Frequently, few details of the fitting procedure are given and the 

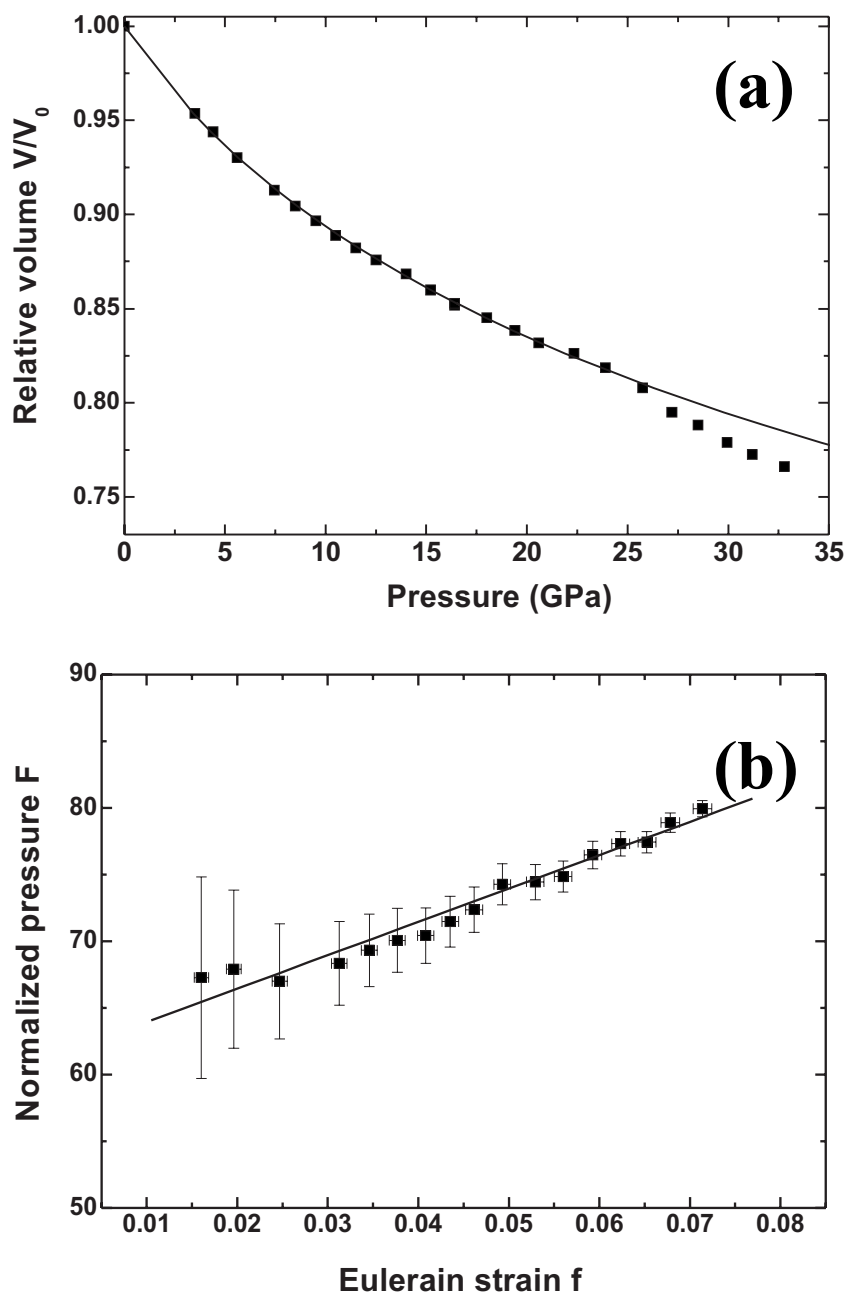

FIG. 3. (a) $V / V_{0}$ plot for $\mathrm{Rb}_{6.15} \mathrm{Si}_{46}$ as a function of pressure (points) along with a third-order Birch-Murnaghan equation of state fitted to the data with $K_{0}=61.4(7) \mathrm{GPa}$ and $K_{0}^{\prime}=6.7(2)$ (solid line). The $K_{0}$ and $K_{0}^{\prime}$ values were obtained from a reduced variable $(F-f)$ plot. (b) Plot of the normalized pressure $(F)$ as a function of the Eulerian strain variable $(f)$ for $\mathrm{Rb}_{6.15} \mathrm{Si}_{46}$.

value of $K_{0}^{\prime}$ assumed or fitted is not quoted. Because $V(P)$ constitutes a nonlinear relation, it is not always easy to design a goodness of fit criterion and to choose the best set of $K_{0}, K_{0}^{\prime}$, and $V_{0}$ parameters. The alternative fitting procedure used here involves transformation of the $V(P)$ data to the normalized or reduced stress-strain variables $F$ and $f$ (Fig. $5) .{ }^{45}$ The Eulerian strain parameter $(f)$ and the normalized pressure $F$ are defined by

$$
\begin{aligned}
& f=\frac{1}{2}\left[\left(\frac{V}{V_{0}}\right)^{-2 / 3}-1\right], \\
& F=P\left[3 f(1+2 f)^{2.5}\right]^{-1} .
\end{aligned}
$$

This formalism yields the second-order finite strain equation

$$
F=K_{0}\left[1-1.5\left(4-K_{0}^{\prime}\right) f\right]
$$

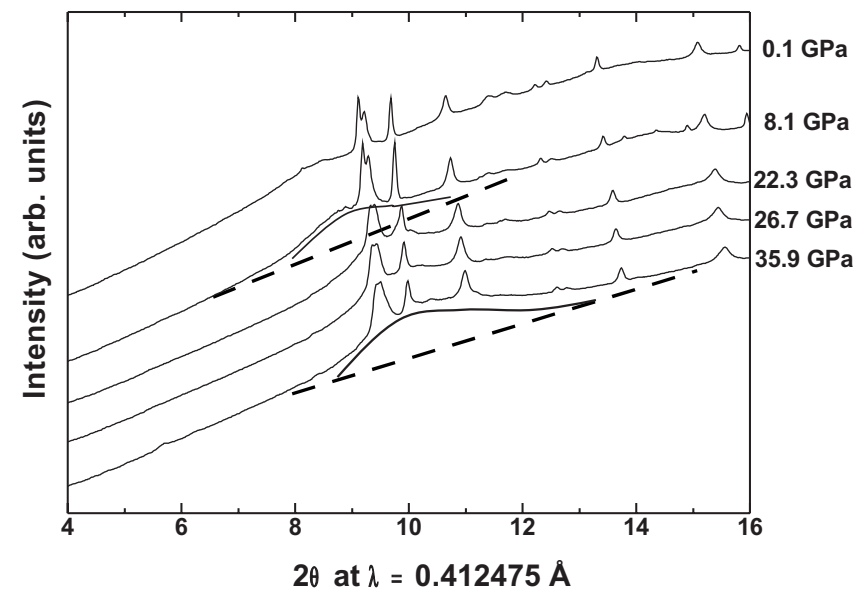

FIG. 4. Selected x-ray diffraction patterns during decompression. The broad feature under the crystalline peaks of $\mathrm{TaN}$ and $\mathrm{TaO}$ are due to the amorphous $\mathrm{Rb}-\mathrm{Si}$ alloy obtained at pressure higher than $33 \mathrm{GPa}$. Dashed lines and solid lines are guides for the eyes, emphasizing the background and the amorphous halo, respectively.

We can then conduct a linear fit to the resulting $F-f$ plot. In that case, well accepted goodness of fit criteria can be applied to the linear fit and likely vs unlikely ranges of values for $K_{0}^{\prime}$ can be readily tested against the data within its error bars. $K_{0}$ and $K_{0}^{\prime}$ were then obtained as the intercept as $f \rightarrow 0$ and the slope of the $F-f$ plot, respectively. This procedure yielded final values of $K_{0}=61.4(7) \mathrm{GPa}$ and $K_{0}^{\prime}$ $=6.7(2)$ for a measured $V_{0}=1088.4 \AA^{3}$.

We also took the opportunity to determine $K_{0}$ and $K_{0}^{\prime}$ for $\mathrm{TaO}$ that has not yet been reported in the literature. Using an $F$ - $f$ plot, the values $K_{0}=293(3) \mathrm{GPa}$ and $K_{0}^{\prime}=5.4(3)$ were obtained for a measured $V_{0}$ of $88.0(2) \AA^{3}$.

The presence of $\varepsilon$ - $\mathrm{TaN}$ and $\mathrm{TaO}$ as internal standards presents within the sample, neither of which undergo any phase transitions throughout the pressure range studied, permitted us to make a more detailed analysis of the x-ray diffraction peak widths and intensities of $\mathrm{Rb}_{6.15} \mathrm{Si}_{46}$ clathrate during pressurization, as well as discriminating between changes intrinsic to the clathrate structure and possible effects due to the sample environment. The three most intense Bragg peaks of $\mathrm{Rb}_{6.15} \mathrm{Si}_{46}$ [(222), (320), and (321)] are situated at $2 \theta$ values of $7.96^{\circ}(1), 8.29^{\circ}(1)$, and $8.60^{\circ}(1)$, respectively, at ambient pressure. The shape of each peak was fitted using a pseudo-Voigt function. In Fig. 5(a) we show the changes in $d$ spacings of these peaks between 0-20 GPa. The slopes can be fitted as two linear regimes with an apparent change of slope at $P \sim 13 \mathrm{GPa}$. At first glance, one could argue that this is not surprising as a nonlinear variation of the $d$ spacings with pressure is expected. However, carrying out a reduced variable $F$ - $f$ analysis for the three reveals a possible discontinuity at $P \sim 13 \mathrm{GPa}$ that is reflected in the behavior of the (320) and (321) reflections [Figs. 5(c) and 5(d)]. That result is corroborated by the Raman-scattering data presented below that reveal a change in the spectra occurring at this pressure. We then compare the width of the clathrate peaks normalized to that of the most intense peak of $\mathrm{TaO}$ (Fig. 6). There is an initial broadening of the clathrate peaks with initial pressurization (that cannot be due to any nonhydro- 

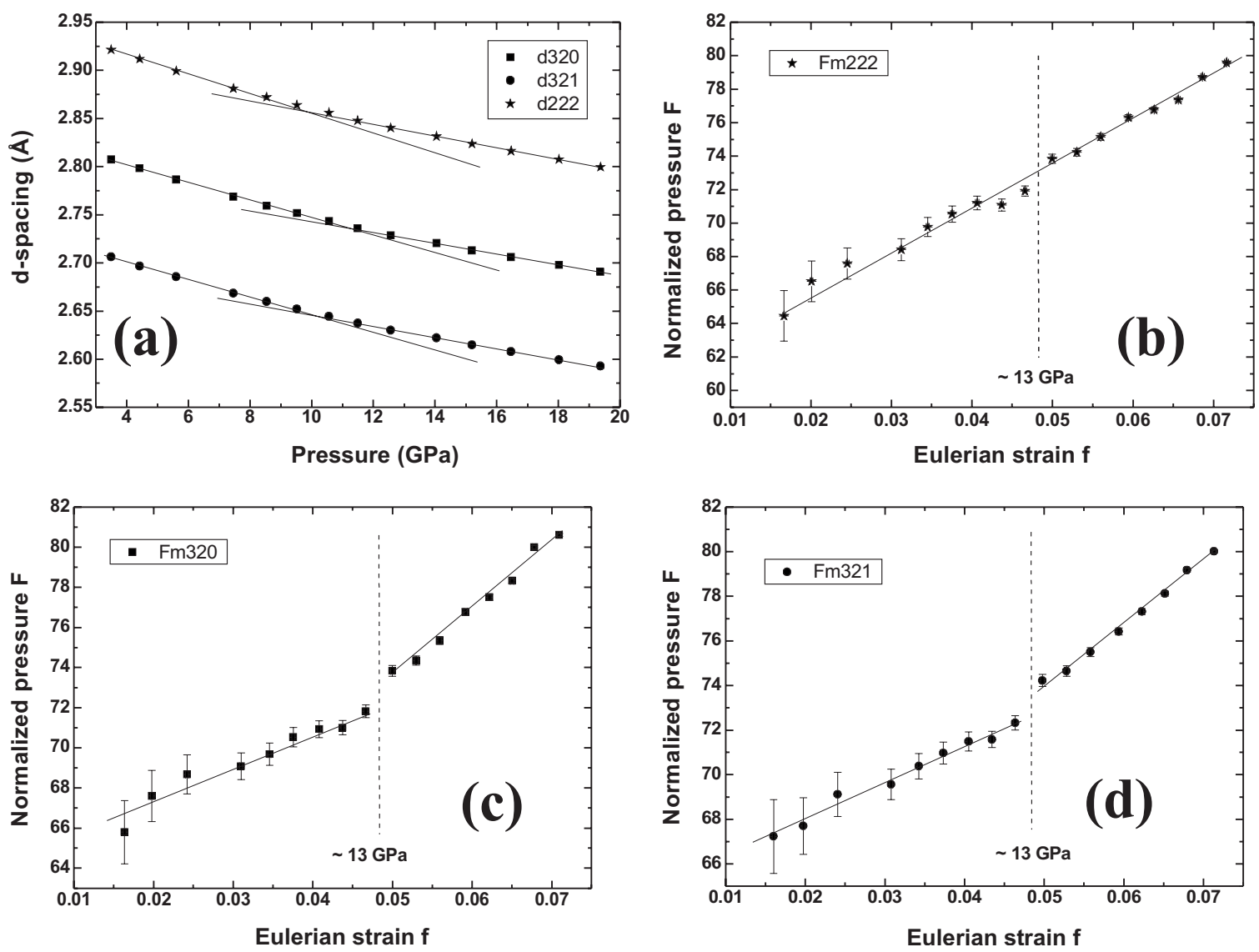

FIG. 5. (a) Change in $d$ spacing for (320), (321), and (222) clathrate reflections with increasing pressure. Two regimes are distinguished with a transition occurring at around $13 \mathrm{GPa}$. (b) Linearized $F-f$ plot based on the data obtained for the (222) peak. (c) $F$ - $f$ plot from the (320) peak data. (d) $F-f$ from the (321) peak data.

static conditions developing within the sample) up to $P$ $\sim 12 \mathrm{GPa}$. Then there is a dramatic increase in the peak width for all three reflections, peaking at $P \sim 13 \mathrm{GPa}$, before returning to the width obtained just before the transition. This significant broadening around 12-13 GPa may be due to the coexistence of two phases with very close cell parameters. This coexistence would exist on a narrow pressure range, either because of the first-order character of the transition or due to the pressure gradient in the volume probed by the X-ray beam. Further pressurization results in a slight decrease in peak width for the (222) and (321) reflections, up to the point of amorphization $(P \sim 30 \mathrm{GPa})$. It is interesting that the "volume collapse" transition observed in the $V(P)$ plot at $P \sim 24 \mathrm{GPa}$ is not accompanied by any observable change in the peak widths of the principal clathrate reflections calibrated using this method. Based on the linewidth observations and also the Raman spectroscopy results described below, we suggest that some structural transformation occurs within the $\mathrm{Rb}_{6.15} \mathrm{Si}_{46}$ clathrate structure at $P$ $\sim 13 \mathrm{GPa}$. These detailed changes are not readily visible on the $V / V_{0}$ plot (Fig. 4). However, they represent a sudden change to some $d_{\mathrm{hkl}}$ values for the clathrate that are not obviously expressed in all of the reflections. We have carried out a detailed examination of the stress-strain relations in the clathrate using $F$ - $f$ relations, first for the three principal reflections then for all 32 peaks used in the global Le Bail refinement, and we can detect a significant change in slope at the transition point (Fig. 5). We have fitted the $F-f$ relations below and above the transition with two linear regions to estimate $K_{0}$ and $K_{0}^{\prime}$ (Table I). We find that the transition at 13 $\mathrm{GPa}$ is associated with a slight $(7.5 \%)$ decrease in $K_{0}$ and a $20 \%$ increase in $K_{0}^{\prime}$; i.e., the clathrate structural modification above the transition range is mechanically "softened" in that it is more compressible and its compressibility changes more rapidly with pressure.

At pressures above the volume collapse $(P>24 \mathrm{GPa})$, data were analyzed using a third-order Birch-Murnaghan equation of state. The fitted values obtained are $V_{0}$ $=1241(80) \AA^{3}, K_{0}=48(18)$, and $K_{0}^{\prime}=2.8(5)$.

In conclusion, our angle-dispersive synchrotron x-ray diffraction studies and subsequent analysis of the data indicate that $\mathrm{Rb}_{6.15} \mathrm{Si}_{46}$ undergoes two phase transformations at $P$ $\sim 13 \mathrm{GPa}$ and $P=24 \pm 1 \mathrm{GPa}$. Both are apparently isostructural transformations in the sense that no additional reflections or peak splittings are observed to indicate a deviation from the $P m \overline{3} m$ cubic clathrate structure. However, a slight distortion of the cubic structure cannot be discarded. The different pressure behaviors of the (222), (320), and (321) $d$ spacings observed in Fig. 5 are not expected to occur in a strictly cubic structure. The first "transition" at $\sim 13 \mathrm{GPa}$ is associated with a marked broadening in the principal (222), (320), and (321) reflections over a narrow pressure range and it corresponds to an abrupt change in the $K_{0}$ and $K_{0}^{\prime}$ values of the clathrate crystal. The second transition is associated with 

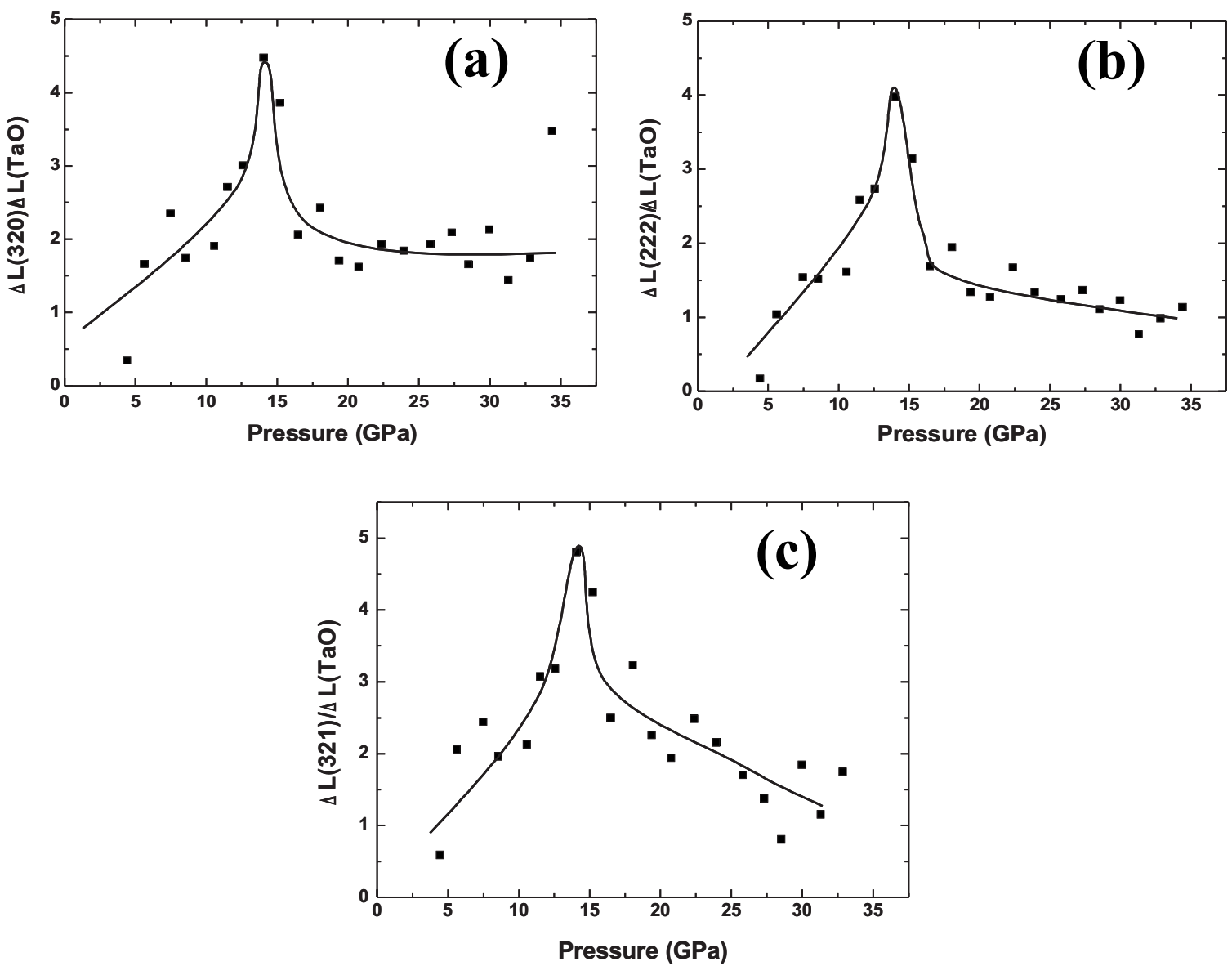

FIG. 6. $\mathrm{TaO}$ is used as an internal standard to estimate intrinsic broadening of the clathrate peak. Ratio of the width of selected $\mathrm{Rb}_{6.15} \mathrm{Si}_{46}$ reflections with respect to the width of the TaO (222) peak: (a) $\mathrm{Rb}_{6.15} \mathrm{Si}_{46}$ (320), (b) $\mathrm{Rb}_{6.15} \mathrm{Si}_{46}$ (222), and (c) $\mathrm{Rb}_{6.15} \mathrm{Si}_{46}(321$ ).

an abrupt change to a more negative slope in the $V(P)$ relation and it seems to correspond to the "volume collapse" transition previously described for $\mathrm{K}_{8} \mathrm{Si}_{46}, \mathrm{Ba}_{8} \mathrm{Si}_{46}$, and $\mathrm{I}_{8} \mathrm{Si}_{44} \mathrm{I}_{2}$ clathrates. 5

Finally, pressure-induced amorphization (PIA) occurs at $33 \pm 1 \mathrm{GPa}$, when the $V / V_{o}$ value has attained $\sim 0.77$ (i.e., $V \sim 838 \AA^{3}$ ). The sample does not show evidence of crystallization into any of the stable or metastable high-pressure phases of $\mathrm{Si}$ (or to produce elemental $\mathrm{Rb}$ ) at high pressure following PIA or during decompression to ambient condi-

TABLE I. $K_{0}$ and $K_{0}^{\prime}$ values obtained from a reduced variable $(F-f)$ plot analysis for the (222), (320), and (321) reflections and from the global LeBail refinement of lattice parameters and unitcell volume. Two regimes were considered: the low-pressure regime up to $13 \mathrm{GPa}$ and the high-pressure regime from 13 to $24 \mathrm{GPa}$.

\begin{tabular}{lccccc}
\hline \hline & Low pressure $(0-13 \mathrm{GPa})$ & & \multicolumn{2}{l}{ High pressure $(13-24 \mathrm{GPa})$} \\
\cline { 2 - 3 } \cline { 5 - 6 } \cline { 5 - 6 } & $\begin{array}{c}K_{0} \\
(\mathrm{GPa})\end{array}$ & $\begin{array}{c}K_{0}^{\prime} \\
(\mathrm{GPa})\end{array}$ & & $\begin{array}{c}K_{0} \\
(\mathrm{GPa})\end{array}$ & $\begin{array}{c}K_{0}^{\prime} \\
(\mathrm{GPa})\end{array}$ \\
\hline$(222)$ & $61.4(6)$ & $6.5(6)$ & & $60.3(8)$ & $6.9(4)$ \\
$(320)$ & $63.8(5)$ & $5.8(6)$ & & $56.5(9)$ & $8.0(5)$ \\
$(321)$ & $64.7(3)$ & $5.7(3)$ & & $60.0(6)$ & $7.1(3)$ \\
Global & $64.0(9)$ & $5.7(9)$ & & $60(1)$ & $7.0(7)$ \\
\hline \hline
\end{tabular}

tions (Fig. 3). We could not carry out any quantitative analysis of the amorphous x-ray diffraction patterns, but the main amorphous diffraction feature was observed to shift to lower $2 \theta$ values as the pressure was released. We investigated the detailed behavior of the amorphous $\mathrm{Rb}_{6.15} \mathrm{Si}_{46}$ material produced via PIA during decompression using Raman spectroscopy, described in Sec. III B.

\section{B. Raman spectroscopy}

The expected Raman-active modes for the clathrate type-I structure are predicted using symmetry analysis. 54 atoms located within the primitive unit cell corresponding to space group $P m \overline{3} n$ (i.e., $\left.\mathrm{O}_{h}^{3}\right)$ give rise to 162 zone-center $(\mathbf{k}=0)$ vibrational modes. Those that are predicted to be Raman active are

$$
\Gamma_{\text {Raman }}=3 A_{1 g}+8 E_{g}+9 T_{2 g} .
$$

Two Raman-active modes of symmetry $E_{g}$ and $T_{2 g}$ are expected to occur at low frequency $\left(<200 \mathrm{~cm}^{-1}\right)$, mainly involving the encapsulated $\mathrm{Rb}$ atoms in the large $(6 d)$ guest sites. $^{46-49}$ These modes are uncoupled from the framework vibrations and they are thought to play an important role in determining the anomalously low lattice thermal conductivity of the clathrate structures. ${ }^{50,51}$ The remaining modes are associated with $\mathrm{Si}-\mathrm{Si}$ stretching and bending vibrations of the 


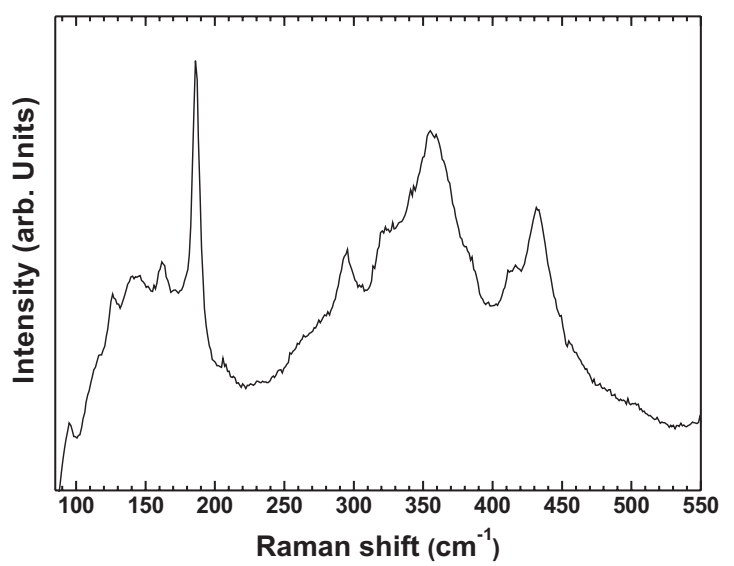

FIG. 7. Raman spectrum of $\mathrm{Rb}_{6.15} \mathrm{Si}_{46}$ measured at $1 \mathrm{~atm}$ and room temperature.

clathrate framework. The Raman spectrum at ambient conditions (Fig. 7) is in good agreement with those previously reported for analogous type-I clathrates (i.e., $\mathrm{K}_{8} \mathrm{Si}_{46}$ and $\left.\mathrm{Ba}_{8} \mathrm{Si}_{46}\right) .{ }^{28}$ Usually, most of Raman bands are broad. In some cases, these broad bands may contain unresolved Raman lines and less Raman bands than expected are observed. In the case of $\mathrm{Rb}_{6.15} \mathrm{Si}_{46}$, our experimentally accessible spectral range is situated above $120 \mathrm{~cm}^{-1}$ and all the observed modes are assumed to be related to the Si framework. Using the calculated frequency by $a b$ initio simulations as a starting model for the Lorentzian line-shape analysis, we obtained a good agreement (within 5\%) with the calculations (Table II).
The Raman spectrum recorded by Fang et al. ${ }^{47}$ for the fully filled clathrate $\mathrm{Na}_{8} \mathrm{Si}_{46}$ is quite different from the spectrum for $\mathrm{Rb}_{6.15} \mathrm{Si}_{46}$ at ambient pressure. The sharp lowfrequency peaks all occur at lower wave number ( $<150 \mathrm{~cm}^{-1}$ ) and the strong massif near $370 \mathrm{~cm}^{-1}$ is absent. It is not yet clear why this is the case. The Raman spectrum of $\mathrm{Ba}_{8} \mathrm{Si}_{46}$ clathrate obtained at ambient pressure is generally similar to those of $\mathrm{K}_{8} \mathrm{Si}_{46}$ and $\mathrm{Rb}_{6.15} \mathrm{Si}_{46}$, but with most of the modes shifted to lower wave number. The linewidths and relative intensities of the Raman modes observed for the metallic type-I clathrates are obviously affected by electronphonon coupling and also by the presence of guest site vacancies (or other crystal defects) that may eliminate $k=0$ selection rules. ${ }^{47}$ The high-frequency modes due to $\mathrm{Si}-\mathrm{Si}$ stretching along with some deformation vibrations of the clathrate framework are generally broadened compared to the low-frequency modes that are mainly associated with guest atom vibrations. We do not yet have a precise understanding of the factors governing the line shape, width, and relative Raman intensities of high- vs low-frequency modes in these semiconductor/metallic clathrate compounds and their relationship to order/disorder phenomena among the framework and guest atom sites and details of the electronic structure.

\section{Quasihydrostatic compression}

During the first series of Raman experiments, argon was used as the pressure-transmitting medium to provide a quasihydrostatic compression. Figure 8 shows Raman spectra of

TABLE II. Experimental and calculated zone-center Raman peak positions and their pressure derivative for $\mathrm{Rb}_{6.15} \mathrm{Si}_{46}$. The experimentally accessible spectral range is situated above $120 \mathrm{~cm}^{-1}$. Some peaks could not be determined under high-pressure conditions.

\begin{tabular}{|c|c|c|c|c|}
\hline Mode symmetry & Frequency (calculated) & Frequency (measured) & $(\partial \nu / \partial P)$ (calculated) & $(\partial \nu / \partial P)$ (measured) \\
\hline$T_{2 g}$ & 72.5 & Not accessible & 1.7 & Not accessible \\
\hline$E_{g}$ & 89.6 & Not accessible & 2.3 & Not accessible \\
\hline$T_{2 g}$ & 122.7 & 126.0 & 0.9 & $0.3(1)$ \\
\hline$E_{g}$ & 138.0 & 141.3 & 2.3 & $0.4(1)$ \\
\hline$T_{2 g}$ & 155.7 & 164.4 & -0.2 & $-0.9(1)$ \\
\hline$T_{2 g}$ & 178.7 & 186.3 & -1.0 & $-1.2(1)$ \\
\hline$E_{g}$ & 203.9 & 207.2 & 3.8 & Not determined \\
\hline$E_{g}$ & 259.1 & 268.0 & 6.1 & Not determined \\
\hline$T_{2 g}$ & 286.4 & 294.9 & 5.5 & $3.1(4)$ \\
\hline$A_{1 g}$ & 314.7 & 320.7 & 5.2 & $3.7(5)$ \\
\hline$E_{g}$ & 333.1 & 337.2 & 10.0 & $12(2)$ \\
\hline$A_{1 g}$ & 344.3 & 353.7 & 11.7 & Not determined \\
\hline$E_{g}$ & 369.2 & 361.1 & 8.5 & Not determined \\
\hline$T_{2 g}$ & 374.8 & 371.6 & 7.3 & Not determined \\
\hline$E_{g}$ & 393.8 & 384.5 & 8.3 & Not determined \\
\hline$E_{g}$ & 399.9 & 398.3 & 7.5 & Not determined \\
\hline$T_{2 g}$ & 403.9 & 413.3 & 6.6 & Not determined \\
\hline$T_{2 g}$ & 415.9 & 431.7 & 7.3 & $5.4(2)$ \\
\hline$A_{1 g}$ & 423.6 & 434.3 & 7.9 & $4.7(2)$ \\
\hline$T_{2 g}$ & 438.5 & 457.7 & 6.2 & Not determined \\
\hline
\end{tabular}




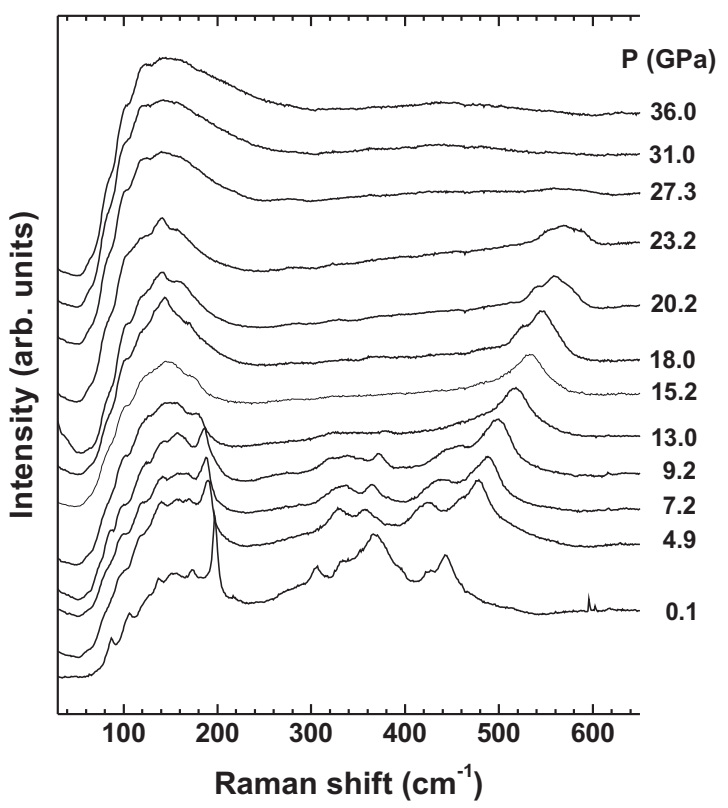

FIG. 8. Raman spectra of $\mathrm{Rb}_{6.15} \mathrm{Si}_{46}$ during quasihydrostatic compression (using argon as pressure-transmitting medium).

$\mathrm{Rb}_{6.15} \mathrm{Si}_{46}$ at selected pressures up to $36 \mathrm{GPa}$ and the pressure dependencies of the Raman frequencies are shown in Fig. 9. During the compression experiments, only nine Raman peaks observed at ambient pressure could be significantly followed.

Between ambient pressure and 9.2 GPa, all the highfrequency peaks $\left(>300 \mathrm{~cm}^{-1}\right)$ shift to higher wave number with increasing pressure. However, the most intense Raman mode initially at $186 \mathrm{~cm}^{-1}$ shifts to lower frequency, as does the weak shoulder at $164 \mathrm{~cm}^{-1}$, indicating some softening of

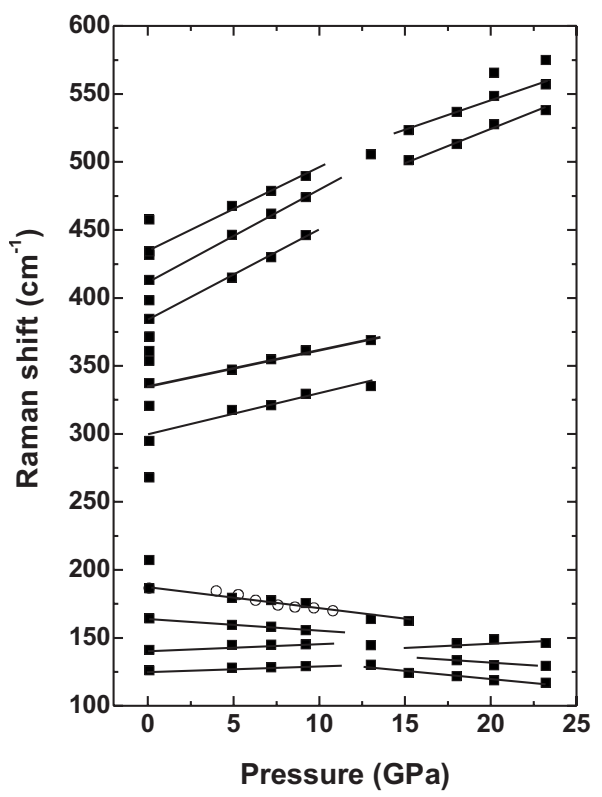

FIG. 9. Raman frequencies of $\mathrm{Rb}_{6.15} \mathrm{Si}_{46}$ plotted as a function of pressure (for quasihydrostatic compression). Lines are drawn as visual aids. Open symbols: Raman frequency of the most intense peak under nonhydrostatic compression. these vibrational modes The other low-frequency modes stiffen slightly with pressure, but much less that the highfrequency vibrations. Similar observations were made for $\mathrm{Ba}_{8} \mathrm{Si}_{46}$ and $\mathrm{K}_{8} \mathrm{Si}_{46}$ type-I clathrates. ${ }^{28,29}$ Kume et al. ${ }^{28}$ noted an analogy with softening in low-lying TA modes at the Brillouin-zone boundary in diamond-structured Si that may be related to the clathrate behavior and could be intrinsic to the $s p^{3}$-bonded tetrahedral lattice. Our ab initio simulations reproduced this behavior in excellent agreement with the experimental results (Table II).

The appearance of the Raman spectrum is affected significantly by increasing pressure. Important changes in the entire spectrum are observed starting from $9.2 \mathrm{GPa}$ but some of the initial peaks can be followed to 13.0-15.2 GPa but already show a strong attenuation above $9.2 \mathrm{GPa}$. Examining of the $d \nu / d P$ pressure variation of modes and their appearance and disappearance, it seems that a likely phase transformation occurs near $13 \mathrm{GPa}$, at the same pressure at which an anomalous broadening in certain $\mathrm{x}$-ray reflections and a mechanical softening of the clathrate lattice are observed. By $18 \mathrm{GPa}$, an entirely new Raman spectrum is recorded, indicating that a change in the clathrate structural parameters affecting the Raman selection rules has occurred. The soft mode initially at $186 \mathrm{~cm}^{-1}$ disappeared and others peaks are replaced by broad bands.

As the pressure is increased, a new peak maximum is identified on the low-frequency shoulder (at $\sim 133 \mathrm{~cm}^{-1}$ ) that has a negative $d \nu / d P$ dependence (Fig. 9). The highfrequency band $\left(\sim 505 \mathrm{~cm}^{-1}\right)$ exhibits a new peak at lower wave number for $P>18 \mathrm{GPa}$ (Fig. 8). These changes in the $\mathrm{Si}-\mathrm{Si}$ stretching vibrations indicate changes in the clathrate crystal structure and the Raman selection rules occurring in the 18-23 GPa range that are associated with the "volume collapse transition" observed in the $V(P)$ relations derived from the synchrotron $\mathrm{x}$-ray diffraction results.

The crystalline peaks are readily observed in the Raman spectrum up to $P \sim 23 \mathrm{GPa}$ and they can hardly be distinguished at $P=27.3 \mathrm{GPa}$ (Fig. 8) after the volume collapse according to x-ray diffraction results. Above $31 \mathrm{GPa}$, the Raman spectrum contains only two very broad and weak bands at $\sim 450$ and $\sim 160 \mathrm{~cm}^{-1}$ that indicate an amorphous structure. This is correlated with the pressure-induced amorphization event indicated by the x-ray diffraction results (Fig. 2).

The amorphous $\mathrm{Rb}_{6.15} \mathrm{Si}_{46}$ Raman spectrum obtained at $36.0 \mathrm{GPa}$ using argon as a pressure-transmitting medium (Fig. 8) closely resembles that of the high-density (HDA) metallic amorphous form of elemental $\mathrm{Si}$ that has atoms in high (fivefold, sixfold) coordination. In amorphous HDA Si, the spectra consist on a single broad feature between 200 and $700 \mathrm{~cm}^{-1}$, with maximum near $400 \mathrm{~cm}^{-1}$. This is similar to the envelope of bands in the vibrational density of states (VDOS) calculated for crystalline silicon with the $\beta$-Sn structure at high pressure and presumably other high-density polymorphs. ${ }^{52,53}$ Our results suggest that pressure destabilization of the $\mathrm{Rb}_{6.15} \mathrm{Si}_{46}$ clathrate, following the volume collapse transition within the clathrate structure, results in formation of a high-density amorphous material with highly coordinated $\mathrm{Si}$ atoms that could be structurally related to the HDA form of elemental $a$-Si. During decompression, the 


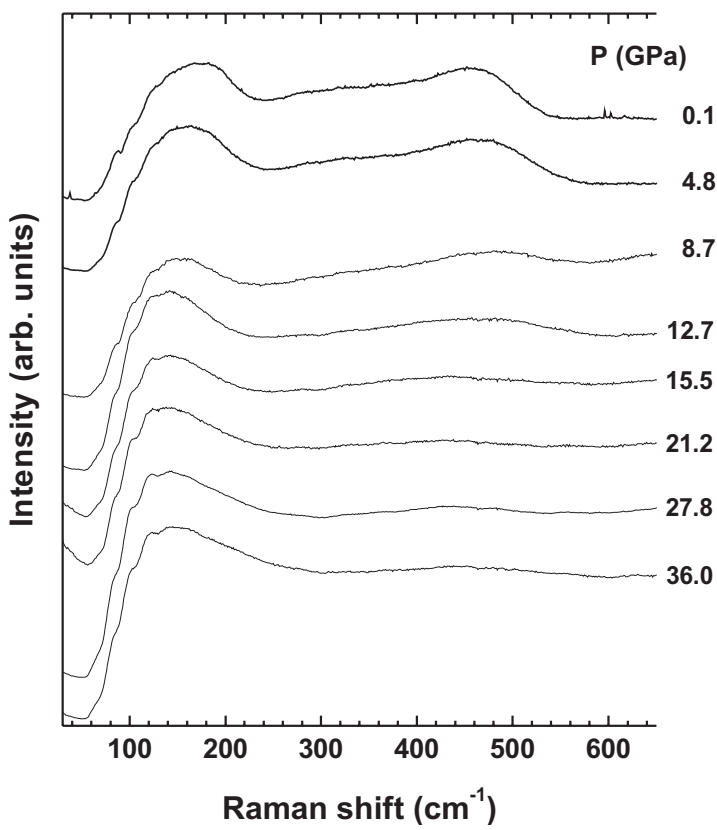

FIG. 10. Raman spectra of $\mathrm{Rb}_{6.15} \mathrm{Si}_{46}$ during decompression after quasihydrostatic compression to $36 \mathrm{GPa}$.

amorphous spectrum changes markedly below $8.7 \mathrm{GPa}$, with the emergence of a broad feature in the Si-Si stretching region at higher wave number $(\sim 460 \mathrm{~cm}-1)$ that is more intense than the previous band of the high-density amorphous form (Fig. 10). The spectrum obtained at ambient conditions resembles that of the tetrahedrally coordinated semiconducting low-density amorphous (LDA) polyamorph of $a-\mathrm{Si}$ dominated by the tetrahedral $\mathrm{Si}-\mathrm{Si}$ stretching vibration at $450 \mathrm{~cm}^{-1}$ and a weaker angle-bending mode at around $300 \mathrm{~cm}^{-1}$. These features reflect a broadened version of the VDOS of the crystalline semiconductor. ${ }^{53}$ Our results indicate that the HDA form of $\mathrm{Rb}_{6.15} \mathrm{Si}_{46}$ produced by PIA from the clathrate has undergone a back transformation into an LDA polyamorphic form during decompression, analogous to the behavior of HDA $a$-Si produced by PIA from nanocrystalline porous-Si or from $a$-Si samples. ${ }^{52,53}$

\section{Nonhydrostatic compression}

It is known that nonhydrostatic stresses can affect the nature and pressure range of crystalline phase transitions or favor pressure-induced amorphization. ${ }^{54,55}$ We submitted $\mathrm{Rb}_{6.15} \mathrm{Si}_{46}$ to a highly nonhydrostatic stress regime by squeezing it without any pressure-transmitting medium. Figure 11 shows the Raman spectra obtained with increasing pressure up to $16.7 \mathrm{GPa}$. The Raman results during compression were comparable to those observed during the hydrostatic compression experiment (Figs. 8 and 11). We observed an expected broadening of the Raman modes (particularly between 250 and $450 \mathrm{~cm}^{-1}$ ). However, the spectrum at 10.5 GPa under nonhydrostatic pressure (Fig. 11) is similar to that obtained at 9.2 GPa during hydrostatic compression (Fig. 8). The pressure dependence of the most intense peak (at $186 \mathrm{~cm}^{-1}$ at ambient pressure) is identical in both cases (Fig. 9). A gradual disappearance of the Raman lines characteristic

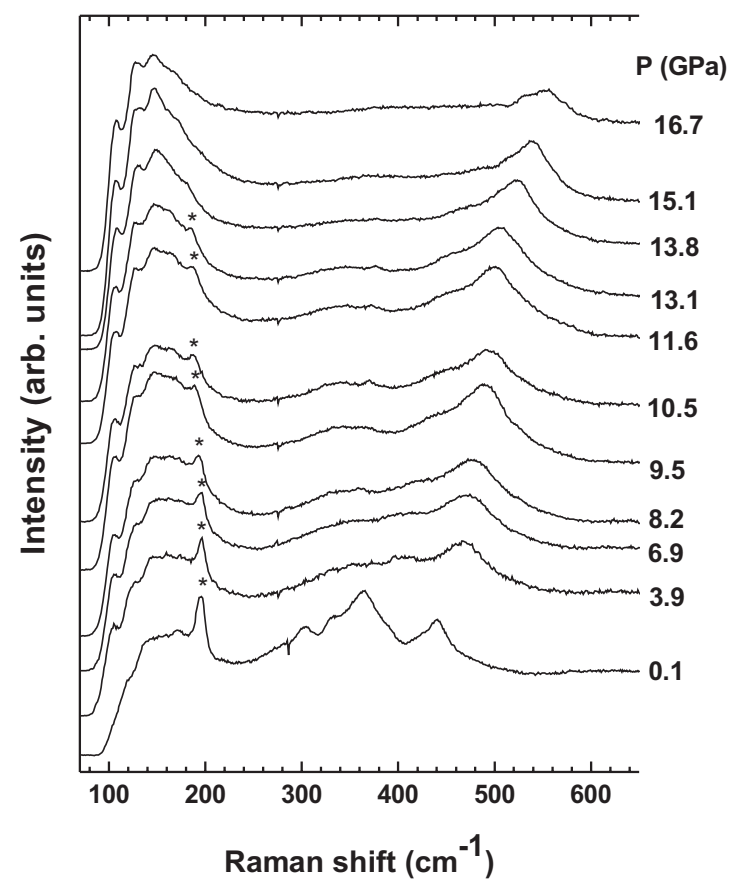

FIG. 11. Raman spectra of $\mathrm{Rb}_{6.15} \mathrm{Si}_{46}$ during a nonhydrostatic compression (no pressure-transmitting medium) to $16.7 \mathrm{GPa}$. Asterisk shows the initially most intense peak at around $190 \mathrm{~cm}^{-1}$.

of the low-pressure phase is observed between 11.6 and 15.1 $\mathrm{GPa}$. The spectrum obtained at $16.7 \mathrm{GPa}$, the highest pressure achieved in that run and that lies lower than the pressure of amorphization, is similar to that obtained during hydrostatic compression at the same pressure. On decompression, we observed a gradual crystal-crystal transformation (Fig. 12). The low-pressure spectra can be interpreted as a superposition of the high-pressure polymorph and the initial lowpressure spectrum, indicating that the crystal structure changes occurring in the 13-18 GPa pressure range could correspond to a first-order phase transition because both phases appear to coexist in the decompressed sample.

\section{DISCUSSION}

Our data for the bulk modulus of $\mathrm{Rb}_{6.15} \mathrm{Si}_{46}$ allow us to further examine the systematics of volume compressibility among Si-framework clathrate-I phases. ${ }^{5}$ One of the striking results of our study is that our value of $K_{0}$ for $\mathrm{Rb}_{6} \mathrm{Si}_{46}$ [61.4(7) $\mathrm{GPa}]$ appears to be much smaller than those reported previously for $\mathrm{K}_{8} \mathrm{Si}_{46}(86 \pm 5 \mathrm{GPa}), \quad \mathrm{Ba}_{8} \mathrm{Si}_{46}$ $(93 \pm 5 \mathrm{GPa}), \mathrm{I}_{8} \mathrm{Si}_{44} \mathrm{I}_{2}(95 \pm 5 \mathrm{GPa})$, or for $(94 \mathrm{GPa})$ or the hypothetical empty cage clathrate $\mathrm{Si}_{46}(87-88 \mathrm{GPa})$ studied theoretically. ${ }^{5,56,57}$ We must first examine whether the large apparent difference in bulk modulus values recorded for the different clathrates reflect real differences in the elastic behavior or if they are due to the details of the various experiments and fitting procedures used to obtain $K_{0}$ from the experimental or theoretical $V(P)$ data. In all of the previous studies, the nonlinear $V(P)$ relation was fit directly using a Murnaghan or Birch-Murnaghan equation of state with $K_{0}^{\prime}$ fixed at 3.6. A detailed analysis based on our data rules this 


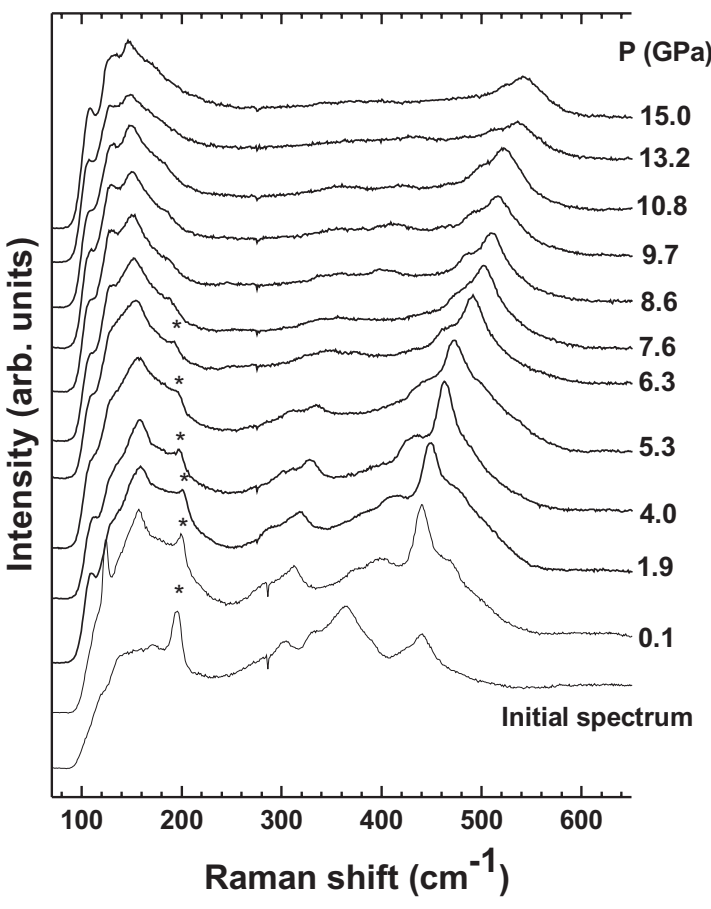

FIG. 12. Raman spectra of $\mathrm{Rb}_{6.15} \mathrm{Si}_{46}$ during decompression after nonhydrostatic compression to $16.7 \mathrm{GPa}$. Asterisk shows the initially most intense peak of the clathrate structure at around $190 \mathrm{~cm}^{-1}$.

out as a viable possibility. If we had fixed $K_{0}^{\prime}$ at 3.6 in our present study of $\mathrm{Rb}_{6.15} \mathrm{Si}_{46}$ clathrate, we would have obtained $K_{0}=78(1) \mathrm{GPa}$, in better apparent agreement with the bulk modulus values reported in previous studies on other type-I clathrates. However, only larger values of $K_{0}^{\prime}$ gave a satisfactory fit to our data; this is especially apparent from considering the linearized $F$ - $f$ plot (Fig. 5; Table I). Recent neutron-diffraction results obtained on $\mathrm{Ba}_{8} \mathrm{Si}_{46}$ compressed with an ethanol-methanol mixture gave $K_{0}=67(2) \mathrm{GPa}$ and $K_{0}^{\prime}=8(1),{ }^{58}$ in close agreement with our values for $\mathrm{Rb}_{6.15} \mathrm{Si}_{46}$. Ab initio simulations on $\mathrm{Ba}_{8} \mathrm{Si}_{46}$ carried out in the present study predict $K_{0}=77 \mathrm{GPa}$ and $K_{0}^{\prime}=4.2$ (Table III). Thus, it appears that $K_{0}$ and $K_{0}^{\prime}$ are highly sensitive to the hydrostaticity conditions. Our conclusion is that the compressibility of $\mathrm{Rb}_{6.15} \mathrm{Si}_{46}$ is larger than $\mathrm{Ba}_{8} \mathrm{Si}_{46}$ or other fully filled clath-

TABLE III. Systematic changes in cell parameter $a_{0}$, the bulk modulus $\left(K_{0}\right)$, and its pressure derivative $\left(K_{0}^{\prime}\right)$ with the progressive filling of the nanocages $(x=0,2,6$, and 8$)$ in the type-I clathrate structure obtained from $a b$ initio calculations.

\begin{tabular}{llll}
\hline \hline & \multicolumn{1}{c}{$a_{0}$} & \multicolumn{1}{c}{$K_{0}$} & \multicolumn{1}{c}{$K_{0}^{\prime}$} \\
\hline $\mathrm{Si}_{46}$ & 10.2296 & 76.055 & 4.0886 \\
$\mathrm{Ba}_{2} \mathrm{Si}_{46}$ & 10.2781 & 77.59 & 4.0516 \\
$\mathrm{Ba}_{6} \mathrm{Si}_{46}$ & 10.3092 & 77.59 & 4.4531 \\
$\mathrm{Ba}_{8} \mathrm{Si}_{46}$ & 10.395 & 76.813 & 4.2288 \\
$\mathrm{Rb}_{2} \mathrm{Si}_{46}$ & 10.28 & 76.185 & 3.9799 \\
$\mathrm{Rb}_{6} \mathrm{Si}_{46}$ & 10.3335 & 74.554 & 4.1928 \\
$\mathrm{Rb}_{8} \mathrm{Si}_{46}$ & 10.4148 & 69.341 & 4.6828 \\
\hline \hline
\end{tabular}
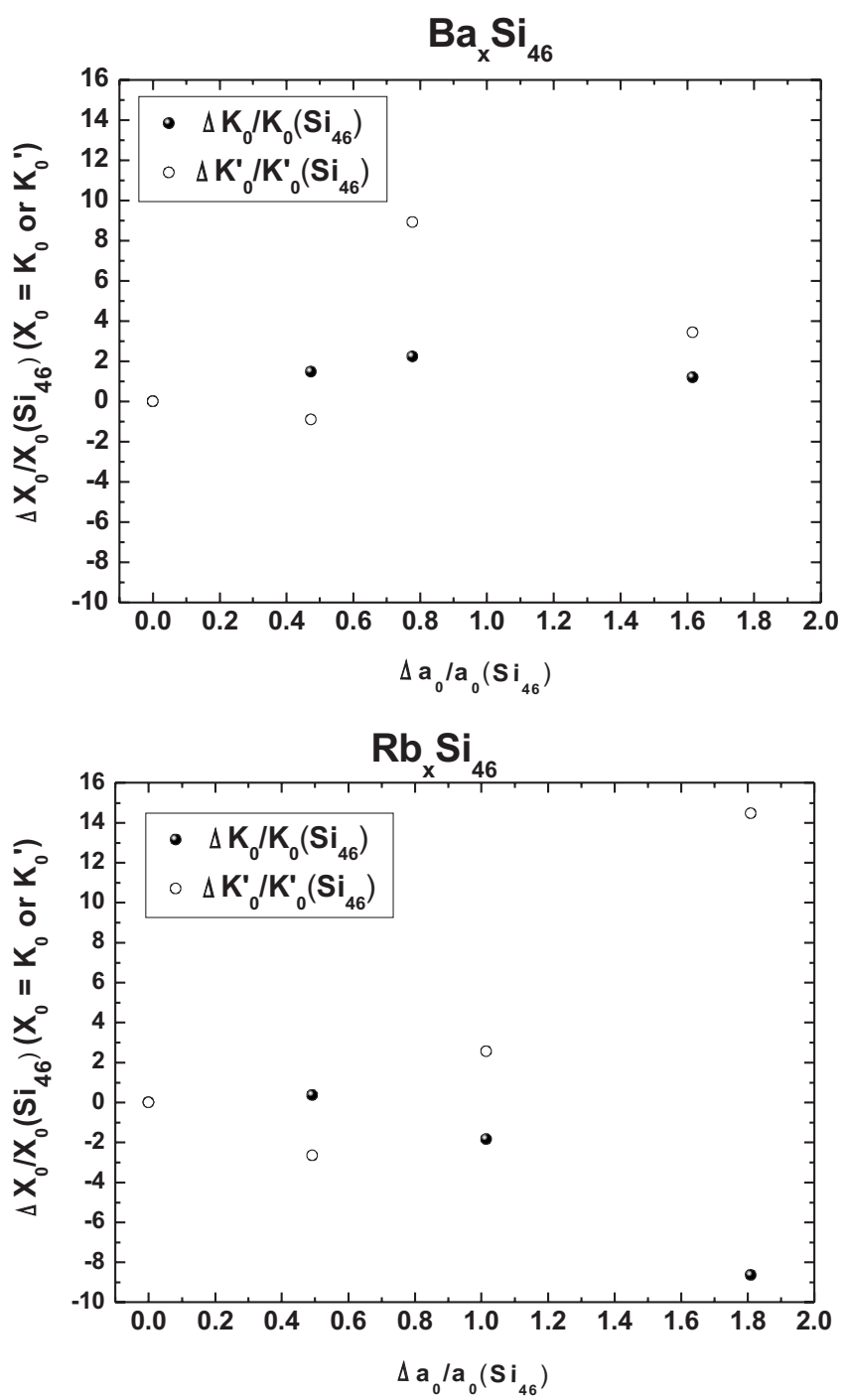

FIG. 13. Ab initio calculated systematic changes in the bulk modulus $\left(K_{0}\right)$ and its pressure derivative $\left(K_{0}^{\prime}\right)$ as a function of the cell parameter $a_{0}$ with progressive filling of the nanocages $(x=0,2$, 6 , and 8) in the type-I clathrate structure. In $\mathrm{Ba}_{x} \mathrm{Si}_{46}$, the effect on $K_{0}$ and $K_{0}^{\prime}$ is negligible. In $\mathrm{Rb}_{x} \mathrm{Si}_{46}$, as soon as the smaller $\mathrm{Si}_{20}$ cages begin to be filled the structure begins to become mechanically weaker.

rates, but to a lesser extent than originally thought by an analysis of the data existing in the literature. We used $a b$ initio methods to calculate the cell parameter, the bulk modulus $K_{0}$, and its derivative $K_{0}^{\prime}$ for $\mathrm{Ba}_{x} \mathrm{Si}_{46}$ and $\mathrm{Rb}_{x} \mathrm{Si}_{46}$ with varying metal cage site occupancies of $x=0,2,6$, and 8 . The results are summarized in Table III and Fig. 13. Filling the cages with guest atoms increases the cell parameter by up to $1.6 \%$ and $1.8 \%$ for $\mathrm{Ba}_{8} \mathrm{Si}_{46}$ and $\mathrm{Rb}_{8} \mathrm{Si}_{46}$, respectively. The mechanical properties are not strongly affected (less than $2-3 \%$ ) by cage occupancy except when the small $\mathrm{Si}_{20}$ cages start to be filled with $\mathrm{Rb}$ atoms. The filling of all the $\mathrm{Si}_{20}$ cages leads to a decrease of $K_{0}$ by $10 \%$ and an increase of $K_{0}^{\prime}$ by $15 \%$. These results clearly show that the presence of $\mathrm{Rb}$ atoms in the $\mathrm{Si}_{20}$ cages deteriorates the mechanical properties of the clathrate. Therefore, the fact that $24 \%$ of the $\mathrm{Si}_{20}$ cages are filled with $\mathrm{Rb}$ atoms in the sample studied here is cer- 
tainly the reason why the bulk modulus is slightly lower than the values recorded for other type-I clathrates.

The analysis of individual peaks in the x-ray diffraction patterns and Raman spectra indicate a phase transition occurring at around $13 \mathrm{GPa}$. As it is shown in Fig. 2, in phase 1 (between ambient $P$ and $\sim 12 \mathrm{GPa}$ ), a progressive reduction of the intensity of these peaks is observed (Fig. 2 inset). These changes could be interpreted as a progressive introduction of disorder into the structure. Disorder due to guest atom displacement with respect to the center of the nanocages has in fact been observed to accompany $\mathrm{Na}$ intercalation in clathrates at room pressure. ${ }^{59}$ Nevertheless, it has been shown that such off-center guest displacement is not compatible with large guest atoms such as $\mathrm{Rb} .{ }^{60}$ Disordering associated with compression of phase 1 should then involve the Si framework. Such distortion of the nanocage framework would be compatible with a loose on the cohesive energy and hence with a lowered value of $K_{0}$ observed at the transition

Raman spectroscopy is a local probe with a short coherence length and is more sensitive to static disorder than X-ray diffraction as illustrated in relaxor materials for instance. ${ }^{61}$ At pressure above $13 \mathrm{GPa}$, the Raman spectra show only broad features. This suggests that a disordered displacement of $\mathrm{Si}$ atoms without change of their average position (accounted by x-ray diffraction) induces a relaxation of the Raman selection rules. The nature of this transition and the induced distortion requires more studies to understand its role on the volume collapse.

Our data on the $V(P)$ relations in $\mathrm{Rb}_{6.15} \mathrm{Si}_{46}$ clathrate show that a homothetic "volume collapse" transition occurs at 24 $\mathrm{GPa}$, analogous to those previously observed for the type-I clathrates $\mathrm{Ba}_{8} \mathrm{Si}_{46}, \mathrm{~K}_{8} \mathrm{Si}_{46}$, and $\mathrm{I}_{6} \mathrm{Si}_{44} \mathrm{I}_{2}$, but not for $\mathrm{Na}_{8} \mathrm{Si}_{46} \cdot{ }^{5,27-31}$ These anomalies in the $V(P)$ relations most likely correspond to an unusual type of symmetry-conserving phase transition. ${ }^{5}$ Various explanations for the transition have been proposed, including the presence of electronic ${ }^{30,62}$ or phonon instabilities, ${ }^{28}$ or possibly vacancy formation on the framework silicon sublattice. ${ }^{63} \mathrm{X}$-ray absorption studies on $\mathrm{Ba}_{8} \mathrm{Si}_{46}$ show a discontinuity in the $\mathrm{Ba} K$-edge position that could indicate a change in electronic structure at the transition. ${ }^{30}$ Tse et al. ${ }^{27}$ suggested an interpretation in which entire phonon branches associated with $K$ guest atom displacements become unstable in $\mathrm{K}_{8} \mathrm{Si}_{46}$ at the transition pressure, resulting in positionally disordered form of the clathrate. Itaka has recently proposed a model in which vacancies could occur on silicon framework sites to yield a defective clathrate $\mathrm{Ba}_{8} \mathrm{Si}_{43}$, analogous to the family of isomorphous $E_{8} \mathrm{Ge}_{43}$ ( $E$ : electropositive element) materials in which where there are always Ge vacancies. ${ }^{63}$ However, recent accurate $\mathrm{x}$-ray measurements performed on $\mathrm{Ba}_{8} \mathrm{Si}_{46}$ in highly hydrostatic conditions did not detect the formation of any amorphous or crystalline high-pressure $\mathrm{Si}$ phases after the collapse. ${ }^{64}$ Other recent results using a combined Rietveld and maximum-entropy approach to analyze the x-ray diffraction data suggest that the volume collapse transition of $\mathrm{Ba}_{8} \mathrm{Si}_{46}$ could be associated with $s-p-d$ rehybridization of the $\mathrm{Si}$ atoms at high pressure, leading to a weakening of the $\mathrm{Si}-\mathrm{Si}$ bonds and a collapse of the unit-cell volume without changing the crystalline structure. ${ }^{62}$ In this interpretation of
Tse et $a l .{ }^{62}$ (and also proposed by San Miguel et $a l .{ }^{30}$ ), the volume collapse can be traced to an electronic topological transition involving a substantial rearrangement of the valence electron distribution accompanied by positional disordering in the $\mathrm{Si}$ sites. The $\mathrm{Si}-\mathrm{Si}$ bonding electrons are removed from the bonding region leading to the weakening of the $\mathrm{Si}-\mathrm{Si}$ bonds, resulting in a displacive but isostructural phase transition taking place with a large volume reduction. The pressure at which the volume collapse occurs should be controlled by the valence number of the cation. $\mathrm{K}_{8} \mathrm{Si}_{46}$ and $\mathrm{Rb}_{6.15} \mathrm{Si}_{46}$ clathrates containing alkali guest atoms exhibit the volume collapse transition at $23 \pm 3$ and $24 \pm 1 \mathrm{GPa}$, respectively, whereas $\mathrm{Ba}_{8} \mathrm{Si}_{46}$ collapses at a significantly lower pressure $13 \pm 2 \mathrm{GPa}^{5}$

It has been also proposed that the collapse transition occurs when the unit-cell volume attains a critical value. ${ }^{26} \mathrm{It}$ occurs for $\mathrm{K}_{8} \mathrm{Si}_{46}$ and $\mathrm{Ba}_{8} \mathrm{Si}_{46}$ clathrates when the unit-cell volume attains 920-930 $\AA^{3} .5,28$ However, in the case of $\mathrm{Rb}_{6.15} \mathrm{Si}_{46}$, the sharp drop in $V(P)$ is only observed when the unit-cell volume is $885-895 \AA^{3}$. However, it is worth noting that the $\mathrm{Rb}_{6.15} \mathrm{Si}_{46}$ unit-cell volume reaches $920-930 \AA^{3}$ in the pressure range $10-15 \mathrm{GPa}$ where a structural instability is observed associated with elastic softening and changes in the Raman spectra. Analogous elastic softening events and potential evidence for a low-pressure structural transformation has also been recorded for $\mathrm{K}_{8} \mathrm{Si}_{46}$ and $\mathrm{Ba}_{8} \mathrm{Si}_{46}$ at $P$ $=6-7 \mathrm{GPa}$, when the volume attains $1010-1035 \AA^{3}$. For $\mathrm{Rb}_{6.15} \mathrm{Si}_{46}$, the lower pressure isostructural transformation might also have the character of a first-order thermodynamic transition because it appears to be associated with a large hysteresis, as indicated by the Raman-scattering data (Fig. 11).

Complete amorphization is observed by $\mathrm{x}$-ray diffraction at higher pressure $(33 \mathrm{GPa})$. The amorphization pressure occurs in a similar pressure range to that recorded for $\mathrm{K}_{8} \mathrm{Si}_{46}$ and $\mathrm{Ba}_{8} \mathrm{Si}_{46}$ (40 \pm 3 and $32 \pm 3 \mathrm{GPa}$, respectively).

As in previous studies of various framework and other materials, the PIA event can be interpreted as resulting from a frustrated first-order transformation of the system or due to an elastic instability. ${ }^{27}$ An important additional question arises in the case of the $M_{x} \mathrm{Si}_{46}$ clathrates, in that they can also exhibit compositional disproportionation into a highpressure dense form of $\mathrm{Si}$ and the alkali or alkaline-earth metal element. This may be the case for $\mathrm{Na}_{8} \mathrm{Si}_{46}$ clathrate, ${ }^{26}$ but instead $\mathrm{K}_{8} \mathrm{Si}_{46}, \mathrm{Ba}_{8} \mathrm{Si}_{46}$, and $\mathrm{Rb}_{6.15} \mathrm{Si}_{46}$ appear to undergo PIA while maintaining constant chemical composition. The result is an amorphous alkali or alkaline-earth-silicon alloy.

The Raman data for $\mathrm{Rb}_{6.15} \mathrm{Si}_{46}$ indicate that the amorphous material produced is in a high-density form, similar to the HDA metallic polyamorph of $a$-Si produced via PIA from nanocrystalline porous-Si or by compressing the lowpressure (LDA) amorphous semiconductor. ${ }^{44,52,53}$ It is worth noting that the predominance of pentagonal rings in the clathrate structure leads some authors to use clathrates as crystalline prototypes of amorphous silicon or germanium. ${ }^{65}$ Recent works combining high-quality x-ray synchrotron sources and molecular dynamics have been used to investigate high-pressure amorphous structures. ${ }^{53,66}$ In addition, pressure-induced amorphization is commonly observed in cagelike crystals. Greaves et al. ${ }^{67-69}$ described a relationship 
between temperature- and pressure-induced amorphization in zeolites accompanied by high-low-density polyamorphic transitions in the resulting amorphous phases. To the best of our knowledge, only little is known about temperature phase diagram of type-I clathrates. These compounds, similar to zeolites, may be expressing similar behavior and further experiments are needed. The observed transformation of highdensity amorphous $\mathrm{Rb}_{6.15} \mathrm{Si}_{46}$ to a low-density polyamorph during decompression indicates that the HDA-LDA transition observed for elemental $a$-Si extends into the binary $\mathrm{Si}-\mathrm{Rb}$ alloy system and presumably into other semiconductor-metal alloy as, for example, in the Zintl phase $\mathrm{BaSi}_{2}$ where pressure-induced amorphization has been observed to occur at around $13 \mathrm{GPa}^{70}$ This observation provides a useful link between studies of PIA and polyamorphism within various compound metalloid systems such as Al-Ge, Ga-Sb, etc. ${ }^{71}$

\section{CONCLUSION}

The type-I clathrate $\mathrm{Rb}_{6.15} \mathrm{Si}_{46}$ at high pressure has been studied by angle-dispersive synchrotron x-ray diffraction, Raman spectroscopy, and ab initio calculations. A phase transition has been observed around $13 \pm 1 \mathrm{GPa}$ from rapid changes occurring in the Raman spectrum and x-ray linewidths, as well as the bulk modulus. A second volumecontracting phase transition occurs around $24 \pm 1 \mathrm{GPa}$, shown by a sudden change in the $V(P)$ relation. This transition may be associated with an electronic topological transition or a phonon instability, resulting in positional disordering of the framework $\mathrm{Si}$ atoms. Pressure-induced amorphization occurs at $33 \pm 1 \mathrm{GPa}$. On decompression, the Raman spectra show changes similar to those observed during the high-density to low-density amorphous transformation in elemental amorphous Si. That result suggests that the HDA-LDA polyamorphic transition extends into the $\mathrm{Si}-\mathrm{Rb}$ system, and the behavior may be general among amorphous semiconductor-metal alloys that do not undergo compositional phase separation.

In summary, the type-I clathrates have a deceptively simple primitive cubic structure that can exhibit a range of unexpected and as yet unexplained transformations during compression, that involve abrupt changes in the cell volume, elastic parameters and vibrational or electronic spectra while maintaining the same average unit-cell symmetry. These changes could arise due to phonon or electronic instabilities involving the host and guest atom sublattices. It is important to study these transitions, because they provide valuable insights into the behavior of these endohedrally doped fullycondensed "fullerane" structures, and they may provide tuning parameters for developing important electronic and thermoelectric or magnetic properties.

\section{ACKNOWLEDGMENTS}

This study was supported by EPSRC-GB under Grant No. EP/D07357X (P.F.M). A.M. and P.R.-H. acknowledge the financial support of the MCYT of Spain under Grants No. MAT2007-65990-C03-03 and No. CSD2007-00045. Part of this research conducted at Oak Ridge National Laboratory's Center for Nanophase Materials Sciences was sponsored by the Scientific User Facilities Division, Office of Basic Energy Sciences, U.S. Department of Energy.

\footnotetext{
*Present address.

${ }^{1}$ C. Cros, M. Pouchard, and P. Hagenmuller, C. R. Hebd. Seances Acad. Sci. 260, 4764 (1965).

${ }^{2}$ J. S. Kasper, P. Hagenmuller, M. Pouchard, and C. Cros, Science 150, 1713 (1965).

${ }^{3}$ C. Cros, M. Pouchard, P. Hagenmuller, and J. S. Kasper, Bull. Soc. Chim. Fr. 7, 2737 (1965).

${ }^{4}$ J. Gallmeier, H. Schäfer, and A. Weiss, Z. Naturforsch. B 24b, 665 (1969).

${ }^{5}$ A. San Miguel and P. Toulemonde, High Press. Res. 25, 159 (2005).

${ }^{6}$ S. Bobev and S. C. Sevov, J. Solid State Chem. 92, 153 (2000).

${ }^{7}$ N. Jaussaud, P. Toulemonde, M. Pouchard, A. San Miguel, P. Gravereau, S. Pechev, G. Goglio, and C. Cros, Solid State Sci. 6, 401 (2004)

${ }^{8}$ G. B. Adams, M. O'Keeffe, A. A. Demkov, O. F. Sankey, and Y.-M. Huang, Phys. Rev. B 49, 8048 (1994).

${ }^{9}$ J. Gryko, P. F. McMillan, R. F. Marzke, G. K. Ramachandran, D. Patton, S. K. Deb, and O. F. Sankey, Phys. Rev. B 62, R7707 (2000).

${ }^{10}$ X. Blase, P. Gillet, A. San Miguel, and P. Mélinon, Phys. Rev. Lett. 92, 215505 (2004).

${ }^{11}$ D. Kahn and J. P. Lu, Phys. Rev. B 56, 13898 (1997).

${ }^{12}$ V. Timoshevskii, D. Connétable, and X. Blase, Appl. Phys. Lett.
}

80, 1385 (2002)

${ }^{13}$ H. Kawaji, H. O. Horie, S. Yamanaka, and M. Ishikawa, Phys. Rev. Lett. 74, 1427 (1995).

${ }^{14}$ S. Yamanaka, E. Enishi, H. Fukuoka, and M. Yasukawa, Inorg. Chem. 39, 56 (2000).

${ }^{15}$ P. Toulemonde, Ch. Adessi, X. Blase, A. San Miguel, and J. L. Tholence, Phys. Rev. B 71, 094504 (2005).

${ }^{16}$ E. D. Sloan, Nature (London) 426, 353 (2003).

${ }^{17}$ Ya. Mudryk, P. Rogl, C. Paul, S. Berger, E. Bauer, G. Hilscher, C. Godart, and H. Noël, J. Phys.: Condens. Matter 14, 7991 (2002).

${ }^{18}$ G. Ramachandran, P. F. McMillan, J. J. Dong, and O. F. Sankey, J. Solid State Chem. 154, 626 (2000).

${ }^{19}$ L.-G. Liu and W. A. Bassett, Elements, Oxides, Silicates: HighPressure Phases with Implications for the Earth's Interior (Oxford University Press, New York, 1986).

${ }^{20}$ G. A. Voronin, C. Pantea, T. W. Zerda, L. Wang, and Y. Zhao, Phys. Rev. B 68, 020102(R) (2003).

${ }^{21}$ S. J. Duclos, Y. K. Vohra, and A. L. Ruoff, Phys. Rev. B 41, 12021 (1990).

${ }^{22}$ A. Ammar, C. Cros, M. Pouchard, N. Jaussaud, J. M. Bassat, G. Villeneuve, M. Dutine, M. Menetrier, and E. Reny, Solid State Sci. 6, 393 (2004).

${ }^{23}$ A. San-Miguel, P. Keghelian, X. Blase, P. Melinon, A. Perez, J. 
P. Itie, A. Polian, E. Reny, C. Cros, and M. Pouchard, Phys. Rev. Lett. 83, 5290 (1999).

${ }^{24}$ G. K. Ramachandran, P. F. McMillan, S. K. Deb, M. Somayazulu, J. Gryko, J. Dong, and O. F. Sankey, J. Phys.: Condens. Matter 12, 4013 (2000).

${ }^{25}$ P. F. McMillan, O. Shebanova, D. Daisenberger, R. Quesada Cabrera, E. Bailey, A. Hector, V. Lees, D. Machon, A. Sella, and M. Wilson, Phase Transitions 80, 1003 (2007).

${ }^{26}$ A. San Miguel, P. Melinon, D. Connetable, X. Blase, F. Tournus, E. Reny, S. Yamanaka, and J. P. Itie, Phys. Rev. B 65, 054109 (2002).

${ }^{27}$ J. S. Tse, S. Desgreniers, Z. Q. Li, M. R. Ferguson, and Y. Kawazoe, Phys. Rev. Lett. 89, 195507 (2002).

${ }^{28}$ T. Kume, T. Koda, S. Sasaki, H. Shimizu, and J. S. Tse, Phys. Rev. B 70, 052101 (2004).

${ }^{29}$ T. Kume, H. Fukuoka, T. Koda, S. Sasaki, H. Shimizu, and S. Yamanaka, Phys. Rev. Lett. 90, 155503 (2003).

${ }^{30}$ A. San Miguel, A. Merlen, P. Toulemonde, T. Kume, S. LeFloch, A. Aouizerat, S. Pascarelli, G. Aquilanti, O. Mathon, T. LeBihan, J. P. Itié, and S. Yamanaka, Europhys. Lett. 69, 556 (2005).

${ }^{31}$ H. Shimizu, T. Kume, T. Kuroda, S. Sasaki, H. Fukuoka, and S. Yamanaka, Phys. Rev. B 68, 212102 (2003).

${ }^{32}$ H.-K. Mao, J. Xu, and P. M. Bell, J. Geophys. Res. 91, 4673 (1986).

${ }^{33}$ A. P. Hammersley, S. O. Svensson, M. Hanfland, A. N. Fitch, and D. Hausermann, High Press. Res. 14, 235 (1996).

${ }^{34}$ E. Soignard and P. F. McMillan, Chem. Mater. 16, 3533 (2004).

${ }^{35}$ P. Hohenberg and W. Kohn, Phys. Rev. 136, B864 (1964); W. Kohn and L. J. Sham, Phys. Rev. 140, A1133 (1965).

${ }^{36}$ G. Kresse, Computer code VASP. See http://cms.mpi.univie.ac.at/ vasp

${ }^{37}$ J. P. Perdew, K. Burke, and M. Ernzerhof, Phys. Rev. Lett. 78, 1396 (1997).

${ }^{38}$ P. E. Blöchl, Phys. Rev. B 50, 17953 (1994); G. Kresse and D. Joubert, ibid. 59, 1758 (1999).

${ }^{39}$ H. J. Monkhorst and J. D. Pack, Phys. Rev. B 13, 5188 (1976).

${ }^{40}$ A. Mujica, A. Rubio, A. Muñoz, and R. J. Needs, Rev. Mod. Phys. 75, 863 (2003).

${ }^{41}$ K. Parlinski, Computer code PHONON. See http://wolf.ifj.edu.pl/ phonon

${ }^{42}$ E. Soignard, O. Shebanova, and P. F. McMillan, Phys. Rev. B 75, 014104 (2007).

${ }^{43}$ J. Rodriguez-Carjaval, Physica B 192, 55 (1993).

${ }^{44}$ D. Daisenberger, M. Wilson, P. F. McMillan, R. Quesada Cabrera, M. C. Wilding, and D. Machon, Phys. Rev. B 75, 224118 (2007).

${ }^{45}$ R. J. Angel, in High-Pressure, High-Temperature Crystal Chemistry, edited by R. M. Hazen and R. T. Downs, Reviews in Mineralogy and Geochemistry (Mineralogical Society of America and the Geochemical Society, Washington, D.C., 2000), Vol. 41, p. 35-60.

${ }^{46}$ E. Reny, A. San-Miguel, Y. Guyot, B. Masenelli, P. Melinon, L. Saviot, S. Yamanaka, B. Champagnon, C. Cros, M. Pouchard, M. Borowski, and A. J. Dianoux, Phys. Rev. B 66, 014532
(2002).

${ }^{47}$ S. L. Fang, L. Grigorian, P. C. Eklund, G. Dresselhaus, M. S. Dresselhaus, H. Kawaji, and S. Yamanaka, Phys. Rev. B 57, 7686 (1998).

${ }^{48}$ G. S. Nolas, C. A. Kendziora, J. Gryko, C. W. Myles, A. Poddar, and O. F. Sankey, J. Appl. Phys. 92, 7225 (2002).

${ }^{49}$ J. S. Tse, T. Iitaka, T. Kume, H. Shimizu, K. Parlinski, H. Fukuoka, and S. Yamanaka, Phys. Rev. B 72, 155441 (2005).

${ }^{50}$ J. Dong, O. F. Sankey, and C. W. Myles, Phys. Rev. Lett. 86, 2361 (2001).

${ }^{51}$ G. S. Nolas, T. J. R. Weakley, J. L. Cohn, and R. Sharma, Phys. Rev. B 61, 3845 (2000).

${ }^{52}$ S. K. Deb, M. Wilding, M. Somayazulu, and P. F. McMillan, Nature (London) 414, 528 (2001).

${ }^{53}$ P. F. McMillan, M. Wilson, D. Daisenberger, and D. Machon, Nature Mater. 4, 680 (2005).

${ }^{54}$ P. Gillet, J. Badro, B. Varrel, and P. F. McMillan, Phys. Rev. B 51, 11262 (1995).

${ }^{55}$ D. Machon, V. P. Dmitriev, P. Bouvier, P. N. Timonin, V. B. Shirokov, and H.-P. Weber, Phys. Rev. B 68, 144104 (2003).

${ }^{56}$ C. A. Perottoni and J. A. H. da Jornada, J. Phys.: Condens. Matter 13, 5981 (2001).

${ }^{57}$ D. Connétable, V. Timoshevskii, E. Artacho, and X. Blase, Phys. Rev. Lett. 87, 206405 (2001).

${ }^{58}$ P. Toulemonde, D. Machon, M. Tucker and A. San Miguel (unpublished).

${ }^{59}$ F. Brunet, P. Mélinon, A. San Miguel, P. Kéghélian, A. Perez, A. M. Flank, E. Reny, C. Cros, and M. Pouchard, Phys. Rev. B 61, 16550 (2000).

${ }^{60}$ H. Libotte, J.-P. Gaspard, A. San Miguel, and P. Mélinon, Europhys. Lett. 64, 757 (2003).

${ }^{61}$ J. Kreisel and P. Bouvier, J. Raman Spectrosc. 34, 524 (2003).

${ }^{62}$ J. S. Tse, R. Flacau, S. Desgreniers, T. Iitaka, and J. Z. Jiang, Phys. Rev. B 76, 174109 (2007).

${ }^{63}$ T. Iitaka, Phys. Rev. B 75, 012106 (2007).

${ }^{64}$ L. Yang, Y. M. Ma, T. Iitaka, J. S. Tse, K. Stahl, Y. Ohishi, Y. Wang, R. W. Zhang, J. F. Liu, H.-K. Mao, and J. Z. Jiang, Phys. Rev. B 74, 245209 (2006).

${ }^{65}$ F. C. Weinstein and E. A. Davis, J. Non-Cryst. Solids 13, 153 (1973).

${ }^{66}$ P. F. McMillan, M. Wilson, M. C. Wilding, D. Daisenberger, M. Mezouar, and G. N. Greaves, J. Phys.: Condens. Matter 19, 415101 (2007).

${ }^{67}$ G. N. Greaves and F. Meneau, J. Phys.: Condens. Matter 16, S3459 (2004).

${ }^{68}$ G. N. Greaves, F. Meneau, O. Marjérus, D. G. Jones, and J. Taylor, Science 308, 1299 (2005).

${ }^{69}$ G. N. Greaves, F. Meneau, A. Sapelkin, L. M. Colyer, I. ap Gwynn, S. Wade, and G. Sankar, Nature Mater. 2, 622 (2003).

${ }^{70}$ T. Nishii, T. Mizuno, Y. Mori, K. Takarabe, M. Imai, and S. Kohara, Phys. Status Solidi B 244, 270 (2007).

${ }^{71}$ E. G. Ponyatovsky and O. I. Barkalov, Mater. Sci. Rep. 8, 147 (1992). 